\title{
Local Observables and Particle Statistics I
}

\author{
SERGIO DOPLICHER \\ Centre Universitaire de Marseille-Luminy \\ RUDOLF HAAG \\ II. Inst. of Theoret. Phys., Univ. Hamburg \\ JOHN E. ROBERTS \\ Dept. of Physics, Princeton University
}

Received July 28, 1971

\begin{abstract}
We consider the family of those states which become asymptotically indistinguishable from the vacuum for observations in far away regions of space. The pure states of this family may be subdivided into superselection sectors labelled by generalized charge quantum numbers. The principle of locality implies that within this family one may define a natural product composition (leading for instance from single particle states to $n$-particle states). Intrinsically associated with the $n$-fold product of states of one sector there is a unitary representation of $P^{(n)}$, the permutation group of $n$ elements, analogous in its role to that arising in wave mechanics from the permutations of the arguments of an $n$-particle wave function. We show that each sector possesses a "statistics parameter" $\lambda$ which determines the nature of the representation of $P^{(n)}$ for all $n$ and whose possible values are $0, \pm d^{-1}$ ( $d$ a positive integer). A sector with $\lambda \neq 0$ has a unique charge conjugate ("antiparticle" states); if $\lambda=d^{-1}$ the states of the sector obey para-Bose statistics of order $d$, if $\lambda=-d^{-1}$ they obey para-Fermi statistics of order $d$. Some conditions which restrict $\lambda$ to \pm 1 (ordinary Bose or Fermi statistics) are given.
\end{abstract}

\section{Introduction}

We continue here our discussion of the superselection structure of elementary particle physics. The setting has been described in some detail in [1] and [2] so a few remarks may suffice here. We consider the net $\mathfrak{A}$ of algebras of local observables ${ }^{1}$ as the basic mathematical object in the theory, and we consider a set $\mathscr{S}$ of states over $\mathfrak{A}$ as representing, in an appropriate idealization, the states of interest in elementary particle physics.

1 This is a correspondence $\mathcal{O} \rightarrow \mathfrak{U}(\mathcal{O})$ between finite regions in space-time and $C^{*}$ algebras. As in [2] we shall always take $\mathcal{O}$ to be a closed double cone (the intersection of a closed forward light cone with a closed backward light cone). The symbol $\mathcal{O}^{\prime}$ denotes the causal complement of $\mathcal{O}$ i.e. the infinitely extended region containing all points spacelike to $\mathcal{O}$. The algebra $\mathfrak{A}\left(\mathcal{O}^{\prime}\right)$ is defined as the $C^{*}$-algebra generated by all $\mathfrak{A}\left(\mathcal{O}_{i}\right)$ with $\mathcal{O}_{i}$ any double cone spacelike to $\mathcal{O}$. The $C^{*}$-algebra generated by all $\mathfrak{A}(\mathcal{O})$ is again denoted by $\mathfrak{A}$. 
Let us discuss first the criterion applied here to characterize $\mathscr{S}$. Recall the connection between states and Hilbert space representations. Each representation has an affiliated family of states, the normal states of the representation (given by vectors and density matrices in the representation space). The pure states affiliated with one irreducible representation form a superselection sector in the sense of [3]; pure states affiliated with inequivalent irreducible representations are separated by a superselection rule. Instead of describing $\mathscr{S}$ we may thus equally well describe the "classes of representations of interest". We do this here by:

1.1. Criterion. Let $\pi_{0}$ be the vacuum representation of $\mathfrak{A}$. We consider as "interesting for elementary particle physics" all representations $\pi$ which are strongly locally equivalent to $\pi_{0}$ in the sense that

$$
\pi\left|\mathfrak{A}\left(\mathcal{O}^{\prime}\right) \cong \pi_{0}\right| \mathfrak{A}\left(\mathcal{O}^{\prime}\right)
$$

for sufficiently many double cones $\mathcal{O}^{2}$. More precisely, $\mathscr{K}_{\pi}$, the set of double cones for which Eq. (1.1) holds, shall contain all translates of some double cone. $\mathscr{S}$ is the set of all states affiliated with the representations satisfying Eq. (1.1).

The physical meaning of the selection criterion 1.1 may be illustrated by the following remark. Take a sequence of increasing double cones $\mathcal{O}_{n}$ which exhaust space-time in the limit $n \rightarrow \infty$ :

$$
\mathcal{O}_{n+1} \supset \mathcal{O}_{n} ; \quad \bigcup_{n} \mathcal{O}_{n}=\boldsymbol{R}^{4}
$$

Then one has for $\omega \in \mathscr{S}$

$$
\lim _{n \rightarrow \infty}\left\|\left.\left(\omega-\omega_{0}\right)\right|_{\mathscr{O}_{n}^{\prime}}\right\|=0
$$

where $\omega_{0}$ denotes the vacuum state and $\left.\phi\right|_{\mathscr{O}_{n}^{\prime}}$ the restriction of a linear form $\phi$ to the subalgebra $\mathfrak{A}\left(\mathcal{O}_{n}^{\prime}\right)$.

The argument leading from criterion 1.1 to Eq. (1.3) will be given in the appendix. There we shall also see how Eq. (1.3), supplemented by some rather natural assumptions, leads back to the criterion 1.1.

Note the close connection between the relationship (1.1) of representations and the asymptotic coincidence of states in the very strong sense of Eq. (1.3). We see from this that the selection criterion 1.1 is too stringent for Quantum Electrodynamics. If it were applied there it would exclude from consideration all states with nonvanishing electric charge because, by Gauss' law, a localized electric charge produces a constant flux of

\footnotetext{
${ }^{2}$ In words: $\pi$ and $\pi_{0}$ when restricted to the algebras of appropriate outer regions shall be unitarily equivalent.
} 
electric field strength through any surrounding sphere, i.e. it gives rise to an effect which is measurable in any $\mathcal{O}_{n}^{\prime}$ and does not decrease as $n \rightarrow \infty$. The selection criterion stated above is aimed only at theories without long range effects, essentially at hadronic physics neglecting electromagnetic or weak interactions. Within this limited regime the criterion appears to be suitable. Thus the states considered in any conventional field theoretical model for hadron physics satisfy the criterion (essentially because they are generated from the vacuum by field operators commuting with the observables at spacelike distances) ${ }^{3}$.

Let us now turn to the structural assumptions on $\mathfrak{A}$ which, together with criterion 1.1, constitute the input of this analysis. First note that as in [2] we may identify the local algebras $\mathfrak{A}(\mathcal{O})$ with weakly closed operator algebras on a Hilbert space $\mathscr{H}_{0}$, the space containing the state vector $\Omega_{0}$ of the vacuum. This is possible without loss of generality because of the local unitary equivalence of all representations of interest. The symbol $\pi_{0}$ then becomes redundant and we shall frequently omit it. Thus

$$
\mathfrak{H}(\mathcal{O})=\pi_{0}(\mathfrak{H}(\mathcal{O}))=\pi_{0}(\mathfrak{H}(\mathcal{O}))^{\prime \prime} \subset \mathscr{B}\left(\mathscr{H}_{0}\right) .
$$

The central assumption of our study is the duality relation in the vacuum representation:

$$
\mathfrak{A}\left(\mathcal{O}^{\prime}\right)^{\prime}=\mathfrak{A}(\mathcal{O}) \quad \text { for each double cone } \mathcal{O} .
$$

It expresses locality and, in addition, the impossibility of enlarging the local observable algebras $\mathfrak{A}(\mathcal{O})$ in the vacuum representation in any way consistent with locality.

The only other assumption used occasionally in the present paper concerns a property which Borchers [4] derived from standard structural assumptions of Quantum Field Theory: ${ }^{4}$

Property $B$. If $E \in \mathfrak{A}(\mathcal{O})$ is a nonzero projection then, for any $\mathcal{O}_{1}$ containing $\mathcal{O}$ in its interior, there is an isometry $W \in \mathfrak{A}\left(\mathcal{O}_{1}\right)$ with $W W^{*}=E$, $W^{*} W=I$ (i.e. within $\mathfrak{A}\left(\mathcal{O}_{1}\right)$ the projection $E$ is equivalent to the identity).

We give now a brief survey of the results and sketch the line of argument.

It is convenient to refer all representations of interest to the Hilbert space $\mathscr{H}_{0}$. Consider one such representation $\pi$ on a Hilbert space $\mathscr{H}_{\pi}$. Due to the "strong local equivalence" of the representations we may choose a double cone $\mathcal{O}$ from $\mathscr{K}_{\pi}$ and a unitary operator $V$ mapping $\mathscr{H}_{0}$

${ }^{3}$ Compare [1], Section VI.

${ }^{4}$ These assumptions themselves, namely Poincaré invariance, positivity of the energy and weak additivity will not be explicitly used in the present paper, although they will be needed in a subsequent finer analysis. 
onto $\mathscr{H}_{\pi}$ so that (on $\mathscr{H}_{0}$ )

$$
\pi(A) V=V A, \quad A \in \mathfrak{U}\left(\mathcal{O}^{\prime}\right) .
$$

Defining then for any $A$

one finds that

$$
\varrho(A)=V^{-1} \pi(A) V
$$

$$
\varrho(A) \in \mathfrak{A} .
$$

It suffices to prove Eq. (1.8) for the case where $A$ belongs to some local algebra $\mathfrak{A}\left(\mathcal{O}_{1}\right)$ with $\mathcal{O}_{1} \supset \mathcal{O}$ since such elements lie dense in $\mathfrak{A}$ in the uniform topology and by the definition (1.7) $\|\varrho(A)\| \leqq\|A\|$. In this case $\varrho(A)$ will commute with $\mathfrak{U}\left(\mathcal{O}_{1}^{\prime}\right)$ and hence, by duality, will belong to $\mathfrak{U}\left(\mathcal{O}_{1}\right)$. Thus

$$
A \rightarrow \varrho(A) \in \mathfrak{U} \subset \mathscr{B}\left(\mathscr{H}_{0}\right)
$$

gives a representation equivalent to $\pi$.

The characteristic properties of the mapping $\varrho$ are:

(i) it maps $\mathfrak{U}$ into $\mathfrak{U}$ preserving the algebraic relations (multiplication, linear combinations and adjoints).

(ii) It is "localized" in some region $\mathcal{O}$ by which we mean that it acts like the identity map in $\mathfrak{U}\left(\mathcal{O}^{\prime}\right)$ (see Eq. (1.6)):

$$
\varrho(A)=A, \quad A \in \mathfrak{U}\left(\mathcal{O}^{\prime}\right) .
$$

In the following we shall call a map with these properties for short a localized morphism of $\mathfrak{A}$ with localization region or support in $\mathcal{O}$. We can sum up the discussion of the last two paragraphs by

1.2. Proposition. The following two conditions are equivalent:

a)

b)

$$
\begin{aligned}
\pi \mid \mathfrak{U}\left(\mathcal{O}^{\prime}\right) & \cong \pi_{0} \mid \mathfrak{U}\left(\mathcal{O}^{\prime}\right) \\
\pi & \cong \pi_{0}{ }^{\circ} \varrho^{5},
\end{aligned}
$$

where $\varrho$ is a localized morphism of $\mathfrak{A}$ with localization region $\mathcal{O}$.

Among localized morphisms there is an obvious equivalence relation: $\varrho_{1} \cong \varrho_{2}$ shall mean that the representations $\pi_{0} \circ \varrho_{1}$ and $\pi_{0} \circ \varrho_{2}$ are unitarily equivalent. We denote the equivalence class of $\varrho$ by $\varrho$. One has

1.3. Lemma ${ }^{6} \cdot \varrho_{1} \cong \varrho_{2}$ if and only if $\varrho_{1}=\sigma \varrho_{2}$ with $\sigma \in \mathscr{I}$.

${ }^{5}$ We have written $\pi_{0} \circ \varrho$ rather than $\varrho$ on the right hand side of (1.11) in order to distinguish the morphism @ from the representation it generates (Eq. (1.9)); the latter results from the composition of the morphism with the defining representation $\pi_{0}$.

${ }^{6}$ As in [2], $\mathscr{I}$ denotes the set of localized inner automorphisms of $\mathfrak{A}$. If $U$ is a unitary element of $\mathfrak{A}$ then $\sigma_{U}$ is the corresponding inner automorphism $\sigma_{U}(A)=U A U^{-1}$. 
The proof of this lemma is omitted since it is analogous to that of Lemma 2.1 in [2].

The morphisms which concern us here have some further properties because the representations of interest satisfy (1.10) not only for one region but for all the translates of it. Thus we define a set $\Delta_{t}(\mathcal{O})$ of morphisms saying that $\varrho$ belongs to $\Delta_{t}(\mathcal{O})$ if it is localized in $\mathcal{O}$ and if for every region resulting from $\mathcal{O}$ by translation there exists a morphism localized there which is equivalent to $\varrho$. The union of the $\Delta_{t}(\mathcal{O})$ as $\mathcal{O}$ varies is denoted by $\Delta_{t}$. Clearly equivalence classes $\Delta_{t} / \mathscr{I}$ are in one-to-one correspondence with the equivalence classes of representations of interest.

The description of representations of interest in the form (1.9) puts into evidence one essential property of this family of representations. They have a natural composition law, corresponding to the multiplication of the respective morphisms. It is easy to see that the product of morphisms respects the class division; in fact we shall find that $\Delta_{t} / \mathscr{I}$ is an Abelian semigroup. The physical picture relating to this structure is the product composition of states which are localized far apart ${ }^{7}$. Take two morphisms $\varrho_{i} \in \Delta_{t}\left(\mathcal{O}_{i}\right), \quad i=1,2$. Correspondingly we have two localized states $\omega_{i}=\omega_{0} \circ \varrho_{i}$ which are vector states in the representations $\pi_{0} \circ \varrho_{i}$. The product state $\omega_{0} \circ \varrho_{1} \varrho_{2}$ is a vector state in the representation $\pi_{0} \circ \varrho_{1} \varrho_{2}$ and has a simple interpretation when $\mathcal{O}_{1}$ and $\mathcal{O}_{2}$ lie spacelike to each other. It is a state which looks like $\omega_{1}$ with respect to observations in $\mathcal{O}_{2}^{\prime}$ and like $\omega_{2}$ for observations in $\mathcal{O}_{1}^{\prime}$.

The family of sectors studied in [2] is a subset of those admitted by our present criterion. Let us call them here simple sectors. A simple sector results from (1.9) if $\varrho$ is an automorphism, i.e. if the image $\varrho(\mathfrak{Q})$ is the whole of $\mathfrak{A}$. Clearly such representations are all irreducible. Taking the direct sum of several such representations one gets a reducible representation which by Lemma 2.5 again satisfies the criterion. Describing the latter in the form (1.9) one is led to a morphism $\varrho$ for which $\varrho(\mathfrak{A}) \neq \mathfrak{A}$. If all $\varrho \in \Delta_{t}$ were to arise in this manner then the consideration of non-automorphic mappings would hardly be of interest. We know, however, from the discussion of the field-theoretical background in Ref. [1] that some nets of observable algebras possess irreducible morphisms which are not automorphisms ${ }^{8}$. This situation arises for instance when the observables are selected from a field algebra by a non-Abelian gauge group. We are primarily interested here in irreducible morphisms and their products.

Consider now an irreducible representation $\pi_{0}{ }^{\circ} \varrho$ and the $n$-fold product composition of this representation with itself. To get a simple physical picture, let us think of states $\omega_{i}=\omega_{0}{ }^{\circ} \varrho_{i}$ with $\varrho_{i} \cong \varrho$ as single

${ }^{7}$ A state $\omega$ is called strictly localized in $\mathcal{O}$ if it coincides with the vacuum for observations in the causal complement $\mathcal{O}^{\prime}$, i.e. if $\left.\left(\omega-\omega_{0}\right)\right|_{\mathscr{O}^{\prime}}=0$.

${ }^{8}$ So $\varrho(\mathfrak{U}) \neq \mathfrak{A}$ but $\varrho(\mathfrak{Q})^{\prime \prime}=\mathfrak{U}^{\prime \prime}=\mathscr{B}\left(\mathscr{H}_{0}\right)$. 
particle states involving the same type of particle and localized in mutually spacelike regions $\mathcal{O}_{i}{ }^{9}$.

Then $\omega_{0}{ }^{\circ} \varrho_{1} \varrho_{2} \ldots \varrho_{n}$ is a state of $n$ identical particles, each localized in a region $\mathcal{O}_{i}$. It is a vector state in the representation $\pi_{0}{ }^{\circ} \varrho^{n}$. We shall see (Lemma 2.7) that this representation is reducible for $n>1$, unless $\varrho$ is an automorphism. Thus unless the single particle states belong to a simple sector there will be many vectors corresponding to the state $\omega_{0}{ }^{\circ} \varrho_{1} \varrho_{2} \ldots \varrho_{n}$. Because, if $V$ is an isometric operator from $\varrho^{n}(\mathfrak{U})^{\prime}$ and $\Psi$ describes this state, then so does $V \Psi$. On the other hand the state vectors $\Psi_{i}$ corresponding to $\omega_{i}$ are unique up to a phase factor and we shall see that, relating each $\varrho_{i}$ to $\varrho$, there is a natural definition of the product of the state vectors so that one may write

$$
\Psi=\Psi_{1} \times \Psi_{2} \times \cdots \times \Psi_{n}
$$

with $\Psi$ corresponding to $\omega_{0} \circ \varrho_{1} \ldots \varrho_{n}$. This product of state vectors is not commutative in contrast to the corresponding commutative product of states. In fact, canonically associated with the representation $\varrho^{n}$ of $\mathfrak{A}$ there is a unitary representation $\varepsilon_{\varrho}^{(n)}$ of the permutation group $P^{(n)}$ commuting with the observables. The operator $\varepsilon_{\rho}^{(n)}(p)$ permutes the order of the state vectors in the product (1.12) by $p \in P^{(n)}$. It is the analogue of the operator in wave mechanics which describes the change of an $n$-particle wave function under this permutation of its arguments. We shall therefore call the collection of (equivalence classes of) the representations $\varepsilon_{\varrho}^{(n)}$ for fixed $\varrho$ and all values of $n$ the statistics of the sector $\hat{\varrho}$. Analyzing the representations in terms of the associated Young tableaux we find that the statistics for $\hat{\varrho}$ is determined by a single number $\lambda$. There are only three possibilities ${ }^{10}$ :

1) $\lambda=d^{-1}, d$ integer. All Young tableaux occur whose columns have length $\leqq d$. This is para-Bose statistics of order $d$.

2) $\lambda=-d^{-1}, d$ integer. All Young tableaux occur whose rows have length $\leqq d$. This is para-Fermi statistics of order $d$.

3) $\lambda=0$, "infinite statistics". All Young tableaux occur without restriction.

9 This should be regarded only as a qualitative picture since strictly localized states in the sense of footnote 7 do not have a sharp particle number.

${ }^{10}$ A similar classification has been given by Hartle, Stolt, and Taylor [5, 6]. Their approach uses as the essential input the cluster property and the existence of "statistics" as postulated in [7] (a pure n-particle state of one particle type is associated with some irreducible representation of $\left.P^{(n)}\right)$. There is also one difference between the classification in [6] and ours. The "infinite order statistics" in [6] includes the mixture of para-Bose and para-Fermi behaviour (compare our Theorem 6.9) which is excluded in our case when $\varrho$ is irreducible. 
Ordinary Bose or Fermi statistics $\lambda= \pm 1$ arises only in the case of simple sectors, i.e. when $\varrho$ is an automorphism. Parastatistics arises in examples constructed as in [1] where the interesting representations of $\mathfrak{A}$ are associated with representations of a compact, non-Abelian gauge group ${ }^{11}$. This structure is reflected in the general setting because we have defined a product on the set of interesting representations of $\mathfrak{A}$ and it turns out that the product of two irreducible representations with finite statistics can be decomposed into a finite direct sum of irreducible representations with finite statistics. This parallels the decomposition of the tensor product of irreducible representations of a compact group. There are no known examples of infinite statistics and it appears hard, if not impossible, to construct such a model even allowing fields with an infinite number of components. But on the level of our present paper we have no arguments against its occurrence.

We denote the set of superselection sectors by $\hat{\mathfrak{A}}_{p}$ (the "physical spectrum" of $\mathfrak{2}$ ) and use the term "charge quantum numbers" for parameters which label the elements of $\hat{\mathfrak{A}}_{p}$. This terminology is not only suggested by looking at the physical significance of $\hat{\mathfrak{A}}_{p}$ in traditional field-theoretical models but can be justified to some extent directly in the present context. We note that the property which distinguishes between states from the sector $\varrho$ and those from the vacuum sector may be localized in any finite region $\mathcal{O} \in \mathscr{K}_{\varrho}$. This "local charge" is an observable property. Shifting such a charge from $\mathcal{O}$ to $\mathcal{O}_{1}$ is an operation which has a welldefined meaning for every state of $\mathscr{S}$. We may apply it for example to the vacuum and thereby obtain a state with the same charge quantum numbers as $\omega_{0}$ but with a local charge of type $\varrho$ in $\mathcal{O}_{1}$ and a compensating "conjugate charge" in $\mathcal{O}$. If we let the region $\mathcal{O}$ move to infinity we arrive at a state in the sector $\hat{\varrho}$. Furthermore, we shall see in Section III that for a sector with finite statistics there is a unique conjugate sector, obtained by this charge transfer operation when we let the region $\mathcal{O}_{1}$ move to infinity. As discussed in [2] the set of simple sectors forms an Abelian group, the product composition corresponding to the ordinary addition of charge quantum numbers and the inverse corresponding to charge conjugation. In general, i.e. for non-simple sectors, the product composition leads to a mixture of charges and so the composition law of charge quantum numbers will be more complicated. Conjugation is now characterized by the fact that composing a sector with its conjugate leads to a mixture of charges one of which has the quantum numbers of the vacuum. An example of a charge quantum number for a non-simple sector is provided by the magnitude of the isospin with the "vector addition model" of isospin as the composition law.

11 The relation of these examples to parafield theory is illustrated in [8]. 


\section{Localized Morphisms}

We note first a few simple facts relating to $\Delta_{t}$ (formulated as Lemmas 2.1 to 2.5 ).

2.1. Lemma. The morphisms from $\Delta_{t}$ are isometries:

$$
\|\varrho(A)\|=\|A\|, \quad \varrho \in \Delta_{t}, A \in \mathfrak{A} .
$$

Hence $\varrho \in \Delta_{t}$ provides an isomorphism of the $C^{*}$-algebras $\mathfrak{A}$ and $\varrho(\mathfrak{A})^{12}$.

Proof. It suffices to prove (2.1) for $A$ localized in some finite region $\mathcal{O}$. By the definition of $\Delta_{t}$ one can then find a morphism $\varrho^{\prime}$ which is both equivalent to $\varrho$ and localized in a region spacelike to $\mathcal{O}$ so that $\varrho^{\prime}(A)=A$ $\varrho(A)=U \varrho^{\prime}(A) U^{-1}=U A U^{-1}$, with $U$ unitary. Taking the norm on both sides one has (2.1).

2.2. Lemma ${ }^{13}$. Let $\varrho \in \Delta_{t}$. The following conditions are equivalent

a) $\varrho$ is an automorphism.

b) The representation @ satisfies the duality relation

$$
\varrho\left(\mathfrak{A}\left(\mathcal{O}^{\prime}\right)\right)^{\prime}=\varrho(\mathfrak{A}(\mathcal{O})), \quad \mathcal{O} \in \mathscr{K}_{\varrho} .
$$

Proof. If $\varrho$ is localized in $\mathcal{O}$ then we have, for the same region $\mathcal{O}$

$$
\varrho\left(\mathfrak{A}\left(\mathcal{O}^{\prime}\right)\right)=\mathfrak{A}\left(\mathcal{O}^{\prime}\right) \text {. }
$$

Since $\varrho(\mathfrak{A}(\mathcal{O}))$ commutes with $\varrho\left(\mathfrak{H}\left(\mathcal{O}^{\prime}\right)\right)$ we get, using duality in the vacuum representation

$$
\varrho(\mathfrak{A}(\mathcal{O})) \subset \varrho\left(\mathfrak{A}\left(\mathcal{O}^{\prime}\right)\right)^{\prime}=\mathfrak{A}\left(\mathcal{O}^{\prime}\right)^{\prime}=\mathfrak{U}(\mathcal{O}) .
$$

If $\varrho$ is an automorphism this may be sharpened to

$$
\varrho(\mathfrak{A}(\mathcal{O}))=\mathfrak{A}(\mathcal{O})
$$

because $\varrho^{-1}$ exists and is localized in the same region so that the above inclusion relation must also hold with $\varrho$ replaced by $\varrho^{-1}$. From equations (2.4), (2.3) and duality in the vacuum representation, we trivially get (2.2) if $\mathcal{O}$ is the localization region of $\varrho$. Now we observe that if the duality relation (2.2) holds for a region $\mathcal{O}$ and a morphism $\varrho$ then it also holds for the same region when $\varrho$ is replaced by any other morphism in the same equivalence class. Hence we have (2.2) whenever there is one morphism in the class $\varrho$ which is localized in $\mathcal{O}$, i.e. as long as $\mathcal{O} \in \mathscr{K}_{\varrho}$. Thus we have proved that condition a) implies b).

Conversely, suppose (2.2) holds for an $\mathcal{O}$ containing the localization region of $\varrho$. Then we can omit $\varrho$ on the left hand side and get, using (1.5),

\footnotetext{
12 Actually, as a consequence of property $B$, the algebra $\mathfrak{A}$ is simple and any representation is isometric [4].

${ }^{13}$ Compare Ref. [1] Theorems 4.1, 5.6, and the note added in proof.
} 
$\varrho(\mathfrak{U}(\mathcal{O}))=\mathfrak{U}(\mathcal{O})$. This means that $\varrho$ becomes an automorphism of such a subalgebra. But, if $\mathscr{K}_{Q}$ contains $\mathcal{O}_{1}$ it also contains any $\mathcal{O}_{2} \supset \mathcal{O}_{1}$. Hence, taking a sequence of increasing double cones $\mathcal{O}_{n}$ as in (1.2), we shall have $\varrho$ acting as an automorphism on $\mathfrak{A}\left(\mathcal{O}_{n}\right)$ for sufficiently large $n$ and we get the statement a).

2.3. Lemma. Morphisms from $\Delta_{t}$ commute when their localization regions are mutually spacelike.

The proof is identical with that of Lemma 2.2 in Ref. [2] just replacing $\Gamma_{c}$ by $\Delta_{t}$.

2.4. Lemma. $\Delta_{t}$ is a semigroup. $\Delta_{t} / \mathscr{I}$ is an Abelian semigroup.

Proof. Given two morphisms $\varrho_{i} \in \Delta_{t}\left(\mathcal{O}_{i}\right)$ the product $\varrho=\varrho_{1} \varrho_{2}$ is a morphism localized in a double cone $\mathcal{O}$ containing $\mathcal{O}_{1}$ and $\mathcal{O}_{2}$. Also, if $\varrho_{i}^{\prime} \cong \varrho_{i}$ then $\varrho^{\prime}=\varrho_{1}^{\prime} \varrho_{2}^{\prime}$ is equivalent to $\varrho$ :

$$
\varrho^{\prime}=\varrho_{1}^{\prime} \varrho_{2}^{\prime}=\sigma_{U_{1}} \varrho_{1} \sigma_{U_{2}} \varrho_{2}=\sigma_{U_{1}} \sigma_{\varrho_{1}\left(U_{2}\right)} \varrho_{1} \varrho_{2}=\sigma_{U_{1} \varrho_{1}\left(U_{2}\right)} \varrho .
$$

This shows on the one hand that $\varrho$ belongs to $\Delta_{t}$ because, choosing $\varrho_{i}^{\prime}$ in (2.5) localized in the region $\mathcal{O}_{i}+x$ resulting from $\mathcal{O}_{i}$ by translation through $x$ (which is possible since $\left.\varrho_{i} \in \Delta_{t}\left(\mathcal{O}_{i}\right)\right)$ we obtain a morphism $\varrho^{\prime}$ localized in $\mathcal{O}+x$ and equivalent to $\varrho$. On the other hand (2.5) also shows that the product respects the class division modulo $\mathscr{I}$ and therefore defines a product of the classes. This product $\hat{\varrho}_{1} \hat{\varrho}_{2}$ is commutative by Lemma 2.3 since we have sufficient freedom in choosing the localization regions of morphisms within one class.

2.5. Lemma. Let $\pi_{1}, \pi_{2}$ be representations satisfying criterion 1.1. Then the direct sum $\pi=\pi_{1} \oplus \pi_{2}$ will also satisfy the criterion and so will any subrepresentation of the $\pi_{i}$.

Proof. This is one of the results for which property B is needed. Take $\pi_{i}(A)=\varrho_{i}(A), i=1,2$ with $\varrho_{i} \in \Delta_{t}\left(\mathcal{O}_{i}\right)$ and choose a sufficiently large $\mathcal{O}$ that contains both $\mathcal{O}_{i}$ in its interior. Then we can find two isometric operators $W_{i} \in \mathfrak{A}(\mathcal{O})$ so that $W_{1}$ maps $\mathscr{H}_{0}$ on a subspace $\mathscr{H}_{1}$ and $W_{2}$ maps $\mathscr{H}_{0}$ on the orthogonal complement $\mathscr{H}_{2}=\mathscr{H}_{1}^{\perp}$. This follows from the fact that every local algebra, being a von Neumann algebra, contains complementary projectors and hence by property $\mathrm{B}$, we have such isometries in a suitable $\mathfrak{A}(\mathcal{O})$. The $W_{i}$ give us a unitary mapping from $\mathscr{H}_{0} \oplus \mathscr{H}_{0}$ onto $\mathscr{H}_{0}$ thereby transforming

$$
\pi(A)=\left(\begin{array}{cc}
\varrho_{1}(A) & 0 \\
0 & \varrho_{2}(A)
\end{array}\right)
$$

into

$$
\varrho(A)=W_{1} \varrho_{1}(A) W_{1}^{*}+W_{2} \varrho_{2}(A) W_{2}^{*} \text {. }
$$


Using the relations

$$
W_{i}^{*} W_{j}=\delta_{i j} I ; \quad \sum_{i=1,2} W_{i} W_{i}^{*}=I
$$

one checks that $\varrho$ is indeed a morphism and that it is localized in $\mathcal{O}$. Clearly the argument goes through when all three regions $\mathcal{O}_{i}, \mathcal{O}$ are shifted by the same translation. Hence $\varrho$ defined by (2.6) belongs to $\Delta_{t}(\mathcal{O})$. It gives us a representation satisfying (1.1) and equivalent to $\pi_{1} \oplus \pi_{2}$.

Consider next the case of a subrepresentation of $\pi_{1}$. It may be described as

$$
\pi_{3}(A)=E \pi_{1}(A)=E \varrho_{1}(A) \text { on the space } \mathscr{H}_{3}=E \mathscr{H}_{0}
$$

where $E$ is a projection commuting with $\varrho_{1}(\mathfrak{Q})$. Since $\varrho_{1}$ acts trivially on $\mathfrak{A}\left(\mathcal{O}_{1}^{\prime}\right)$ we have by duality $E \in \mathfrak{A}\left(\mathcal{O}_{1}\right)$. Hence by property B, there is an isometry $W \in \mathfrak{A}(\mathcal{O})$ mapping $\mathscr{H}_{0}$ onto $\mathscr{H}_{3}$, i.e. satisfying $W W^{*}=E$. The representation $\varrho_{3}$ defined on the space $\mathscr{H}_{0}$ by

$$
\varrho_{3}(A)=W^{*} \varrho_{1}(A) W
$$

is then equivalent to $\pi_{3}$ defined on $\mathscr{H}_{3}$ by $(2.8)$ and one easily checks that $\varrho_{3} \in \Delta_{t}(\mathcal{O})$. This concludes the proof of Lemma 2.5.

The next lemma provides the basis for the discussion of permutation symmetry (statistics). It generalizes Lemma 2.3 of Ref. [2].

2.6. Lemma. Let $\varrho_{1}, \varrho_{2}, \varrho$ be equivalent morphisms from $\Delta_{t}$ so that

$$
\varrho_{i}=\sigma_{U_{i}} \varrho, \quad i=1,2
$$

and let the localization region of $\varrho_{1}$ be spacelike to that of $\varrho_{2}$. Then the unitary operator

$$
\varepsilon_{\varrho}=\varrho\left(U_{2}^{-1}\right) U_{1}^{-1} U_{2} \varrho\left(U_{1}\right)
$$

has the following properties

a) It depends only on $\varrho$ and not on the $\varrho_{i}$ nor the choice of $U_{i}$ (as long as the $\varrho_{i}$ vary within the class $\hat{\varrho}$ and their supports remain spacelike to each other).

b) If $\varrho^{\prime}=\sigma_{W} \varrho$ then

$$
\varepsilon_{\varrho^{\prime}}=\sigma_{V} \varepsilon_{\varrho} \quad \text { with } \quad V=W \varrho(W) .
$$

c) $\varepsilon_{\varrho}$ commutes with all observables in the representation $\varrho^{2}$ i.e.

d)

$$
\varepsilon_{\varrho} \in \varrho^{2}(\mathfrak{H})^{\prime} .
$$


Proof. d) follows from a) because $\varepsilon_{\varrho}^{-1}$ results from $\varepsilon_{\varrho}$ by interchanging $U_{1}$ and $U_{2}$ while according to a) this leaves $\varepsilon_{o}$ unchanged. The statements a), b), c) are verified by straightforward computation. Let us compute the change in $\varepsilon_{\varrho}$ if we replace $\varrho_{i}$ by $\varrho_{i}^{\prime}$ with

$$
\varrho_{i}^{\prime}=\sigma_{U_{i}^{\prime}} \varrho=\sigma_{W_{i}} \varrho_{i} ; \quad U_{i}^{\prime}=W_{i} U_{i} .
$$

We consider only a small change in the support regions so that the supports of $\varrho_{1}, \varrho_{1}^{\prime}, W_{1}$ are spacelike to those of $\varrho_{2}, \varrho_{2}^{\prime}, W_{2}$. Then we have

$$
\varrho_{1}\left(W_{2}\right)=W_{2} ; \quad \varrho_{2}\left(W_{1}\right)=W_{1} ; \quad W_{1} W_{2}=W_{2} W_{1} .
$$

Replacing $U_{i}$ by $U_{i}^{\prime}$ in (2.11) we get

$$
\varepsilon_{\varrho}^{\prime}=\varrho\left(U_{2}^{-1}\right) \varrho\left(W_{2}^{-1}\right) U_{1}^{-1} W_{1}^{-1} W_{2} U_{2} \varrho\left(W_{1}\right) \varrho\left(U_{1}\right) .
$$

Now $U_{2} \varrho\left(W_{1}\right)=\varrho_{2}\left(W_{1}\right) U_{2}=W_{1} U_{2}$ and similarly $\varrho\left(W_{2}^{-1}\right) U_{1}^{-1}=U_{1}^{-1} W_{2}^{-1}$. This together with the commutativity of the $W_{i}$ gives

$$
\varepsilon_{\varrho}^{\prime}=\varepsilon_{\varrho} .
$$

Since any pair of mutually spacelike double cones $\mathcal{O}_{3}, \mathcal{O}_{4}$ can be reached from $\mathcal{O}_{1}, \mathcal{O}_{2}$ by a sequence of small deformations and shifts we obtain the result a) by repeated use of (2.17).

We shall not reproduce here the simple calculations checking (2.12) and (2.13). The definitions and relations involved in Lemma 2.6 will be generalized and become transparent in Section IV.

2.7. Proposition. If $\varrho \in \Delta_{t}$, then the following three conditions are equivalent:

a) $\varrho$ is an automorphism,

b) $\varrho^{2}$ is irreducible,

c) $\varepsilon_{\varrho}= \pm I$.

Proof. The step from a) to b) is trivial; c) follows from b) by (2.13) and (2.14). We still have to show that c) implies a). Taking $\varrho_{2}=\varrho, U_{2}=I$ in Lemma 2.6 we get for $\varepsilon_{Q}$ the expression

$$
\varepsilon_{\varrho}=U_{1}^{-1} \varrho\left(U_{1}\right) .
$$

Let $A \in \mathfrak{A}(\mathcal{O})$ and choose $\varrho_{1}$ so that its support is spacelike to the supports of $A$ and $\varrho$. Then

$$
A=\varrho_{1}(A)=U_{1} \varrho(A) U_{1}^{*} .
$$

If $\varepsilon_{\varrho}= \pm I$ we have by (2.18) $U_{1}= \pm \varrho\left(U_{1}\right)$. Hence $A=\varrho\left(U_{1} A U_{1}^{*}\right)=\varrho(B)$ with $B \in \mathfrak{A}$. Thus any $A \in \mathfrak{A}$ which is localized in some finite region is in the set $\varrho(\mathfrak{H})$, so $\varrho(\mathfrak{U})$ is dense in $\mathfrak{U}$ and, being closed in the norm topology, $\varrho(\mathfrak{A})=\mathfrak{A}$. Hence $\varrho$ is an automorphism. 


\section{Charge Transfer and Charge Conjugation}

Our next objective is to show that all morphisms $\varrho \in \Delta_{t}$ can be obtained as limits of sequences of inner automorphisms. This will allow us to construct a left inverse for every such morphism. It will also suggest a simple physical interpretation in terms of charge transfer and charge conjugation.

Let $\varrho \in \Delta_{t}(\mathcal{O})$ and take a sequence of equivalent morphisms $\varrho_{k} \in \Delta_{t}\left(\mathcal{O}_{k}\right)$ whose respective localization regions move to infinity so as to become eventually spacelike to any given double cone.

Then there is a corresponding sequence of unitaries

$$
U_{k} \in\left\{\mathfrak{A}\left(\mathcal{O}_{k}^{\prime}\right) \cap \mathfrak{A}\left(\mathcal{O}^{\prime}\right)\right\}^{\prime}
$$

such that

$$
\varrho_{k}(A)=\sigma_{U_{k}} \varrho(A)=U_{k} \varrho(A) U_{k}^{-1} .
$$

We know that for any $A \in \mathfrak{A}$

Hence we have

$$
\lim _{k \rightarrow \infty}\left\|\varrho_{k}(A)-A\right\|=0 .
$$

3.1. Lemma. Let $U_{k}$ be defined as in (3.2). Then

$$
\varrho(A)=\lim _{k \rightarrow \infty} \sigma_{U_{k}^{*}}(A)=\lim _{k \rightarrow \infty} U_{k}^{*} A U_{k}
$$

where the convergence is understood in the uniform topology.

We may interpret this in the following way. $\varrho_{k}$ creates a certain type of charge in the region $\mathcal{O}_{k}$. By this we mean that the state $\omega_{0}{ }^{\circ} \varrho_{k}$ is localized in $\mathcal{O}_{k}$ and has the charge quantum numbers of the class $\hat{\varrho}$. In the same sense $\sigma_{U_{k}}$ transfers this type of charge from region $\mathcal{O}$ to $\mathcal{O}_{k}$ and $\sigma_{U_{k}^{k}}$ transfers it from $\mathcal{O}_{k}$ to $\mathcal{O}$. As $k \rightarrow \infty$ the effect within the region $\mathcal{O}_{k}$ may be ignored and hence we get (3.4). The creation of charge in $\mathcal{O}$ has been simulated by the transfer of charge from a far away region to $\mathcal{O}$. This suggests that the inverse sequence $\left(U_{k}\right.$ instead of $\left.U_{k}^{*}\right)$ might lead us in the limit to a charge conjugate state localized in $\mathcal{O}$. Of course if $\varrho$ is an automorphism this limiting procedure is covered by Lemma 3.1, but we shall see that even in the general case this expectation can be justified to some extent.

For this purpose one first observes that the $\sigma_{U}$ belong to a compact subset of a certain topological space. Consider the space $\mathscr{M}$ of bounded linear mappings from $\mathfrak{U}$ into $\mathscr{B}\left(\mathscr{H}_{0}\right)$, equipped with the so-called pointweak-open topology. In this topology the convergence of a sequence (or net) $\phi_{\alpha} \in \mathscr{M}$ to $\phi$ means that for every $A \in \mathfrak{U}$ the sequence (or net) of operators $\phi_{\alpha}(A)$ converges to $\phi(A)$ in the weak operator topology of 
$\mathscr{B}\left(\mathscr{H}_{0}\right)$. The unit ball of

$$
\mathscr{M}\left(\text { i.e., the set } \mathscr{M}_{1}=\left\{\phi \in \mathscr{M}:\|\phi\|=\sup \frac{\|\phi(A)\|}{\|A\|} \leqq 1\right\}\right)
$$

is compact in this topology (compare [9]). This may be used to construct a left inverse for a morphism $\varrho$. It will be convenient to adopt

3.2. Definition. A positive linear mapping $\phi \in \mathscr{M}$ is called a left inverse of the localized morphism @ if

$$
\begin{aligned}
\phi(A \varrho(B)) & =\phi(A) B, \quad A, B \in \mathfrak{A}, \\
\phi(\varrho(A) B) & =A \phi(B), \quad A, B \in \mathfrak{A}, \\
\phi(I) & =I .
\end{aligned}
$$

One checks easily that $\phi$ has the same localization region (support) as $\varrho$ : if $\varrho \in \Delta_{t}(\mathcal{O})$ then

$$
\phi(A)=A \quad \text { for } \quad A \in \mathfrak{A}\left(\mathcal{O}^{\prime}\right) ; \phi\left(\mathfrak{A}\left(\mathcal{O}_{1}\right)\right) \subset \mathfrak{A}\left(\mathcal{O}_{1}\right) \text { when } \quad \mathcal{O}_{1} \supset \mathcal{O} .
$$

Consequently $\phi$ maps $\mathfrak{U}$ into $\mathfrak{A}$. One has

3.3. Lemma. Let $U_{k}$ be defined as in (3.2). Then the sequence $\sigma_{U_{k}}$ possesses at least one limit point in $\mathscr{M}$. Every limit point of this sequence is a left inverse of $\varrho$.

Proof. The first claim follows from $\sigma_{U} \in \mathscr{M}_{1}$ and the compactness of $\mathscr{M}_{1}$. According to Lemma 3.1 the sequence of operators $\sigma_{U_{k}} \varrho(A)$ converges uniformly to $A$.

Therefore

$$
\left\|U_{k} \varrho(A) U_{k}^{*}-A\right\| \rightarrow 0, \quad A \in \mathfrak{A} .
$$

$$
\left\|U_{k} \varrho(A) B U_{k}^{*}-A U_{k} B U_{k}^{*}\right\| \rightarrow 0 ; \quad A, B \in \mathfrak{A} .
$$

Taking an $\mathscr{M}$-convergent subnet of the sequence $\sigma_{U_{k}}$ and denoting its limit by $\phi$ we get (3.6). In an analogous way one obtains (3.5). Each $\sigma_{U}$ maps positive operators into positive ones and the weak limit of a sequence of positive operators is positive. Hence $\phi$ is a positive mapping.

3.4. Lemma. The set of all left inverses of a given $\varrho \in \Delta_{t}$ is a nonvoid, compact, convex subset of the locally convex space $\mathscr{M}$.

Proof. One only has to check that the conditions defining a left inverse for $\varrho$, viz (3.5) to (3.7) and positivity, are stable under taking limits and convex combinations.

3.5. Lemma. Let $\phi$ be a left inverse for $\varrho \in \Delta_{t}$. The cyclic representation of $\mathfrak{A}$ arising from the GNS construction with the state $\omega_{0}^{\circ} \phi$ will be denoted by $\pi_{\phi}$, the cyclic vector by $\xi$, and the representation space by $\mathscr{H}$. 
There is an isometric mapping $V$ from $\mathscr{H}_{0}$ onto a subspace $\mathscr{H}_{1} \subset \mathscr{H}$ such that

$$
\begin{aligned}
V \Omega & =\xi, \\
\pi_{\phi}(\varrho(A)) V & =V A, \\
V^{*} \pi_{\phi}(A) V & =\phi(A),
\end{aligned}
$$

i.e., the representation $\pi_{\phi}{ }^{\circ} \varrho$ contains a subrepresentation (on $\mathscr{H}_{1}$ ) equivalent to $\pi_{0}$ and the matrix elements of $\pi_{\phi}(A)$ in the subspace $\mathscr{H}_{1}$ coincide with the corresponding ones of $\phi(A)$ in $\mathscr{H}_{0}{ }^{14}$.

Proof. Restricting the GNS construction to the subalgebra $\varrho(\mathfrak{A})$ we get a subspace $\mathscr{H}_{1} \subset \mathscr{H}$ in which the vectors $\pi_{\phi}(\varrho(A)) \xi$ are a dense set. The mapping $V$ is then explicitly given by

It is isometric since

$$
V: A \Omega \rightarrow \pi_{\phi}(\varrho(A)) \xi .
$$

$$
\left\|\pi_{\phi}(\varrho(A)) \xi\right\|^{2}=\left(\xi, \pi_{\phi} \varrho\left(A^{*} A\right) \xi\right)=\omega_{0}\left(\phi \varrho\left(A^{*} A\right)\right)=\omega_{0}\left(A^{*} A\right)=\|A \Omega\|^{2}
$$

and it maps $\mathscr{H}_{0}$ onto $\mathscr{H}_{1}$. This is the content of (3.11), (3.12). To check (3.13) we compute the matrix element of the left hand side between vectors of $\mathscr{H}_{0}$. By (3.14) we get

$$
\begin{aligned}
\left(C \Omega, V^{*} \pi_{\phi}(A) V B \Omega\right) & =\left(\pi_{\phi}(\varrho(C)) \xi, \pi_{\phi}(A) \pi_{\phi}(\varrho(B)) \xi\right) \\
& =\omega_{0}\left(\phi\left(\varrho\left(C^{*}\right) A \varrho(B)\right)\right)=\omega_{0}\left(C^{*} \phi(A) B\right) \\
& =(C \Omega, \phi(A) B \Omega)
\end{aligned}
$$

for all $A, B, C \in \mathfrak{A}$ and this proves (3.13).

3.6. Corollary. If $\phi$ is a left inverse then

$$
\phi\left(A^{*} A\right) \geqq \phi\left(A^{*}\right) \phi(A), \quad A \in \mathfrak{A} .
$$

Proof. Let $E_{1}=V V^{*}$ denote the projection on $\mathscr{H}_{1}$ in Lemma 3.5. We get from (3.13)

$$
\phi\left(A^{*} A\right)=V^{*} \pi_{\phi}\left(A^{*}\right) \pi_{\phi}(A) V \geqq V^{*} \pi_{\phi}\left(A^{*}\right) E_{1} \pi_{\phi}(A) V=\phi\left(A^{*}\right) \phi(A) .
$$

Comments. The state $\omega_{0} \circ \phi$ satisfies (1.3) because $\phi$ is localized. Hence, under the circumstances described in the appendix, the representation $\pi_{\phi}$ will satisfy criterion 1.1 and we may then put $\pi_{\phi}(A) \cong \bar{\varrho}(A)$ where $\varrho$ is a localized morphism. In any case, the representations $\pi_{\phi}$ and $\varrho$ can be considered as "charge conjugates", since the composition $\pi_{\phi}{ }^{\circ} \varrho$ leads to a representation containing the vacuum sector. We shall see, that if $\varrho$ is irreducible and has finite statistics then $\phi$ is unique and $\pi_{\phi}$ is irreducible, i.e. $\varrho$ then has a unique charge conjugate sector.

\footnotetext{
${ }^{14}$ Note that (3.13) means that the mapping $\phi$ is "completely positive" in the sense of Stinespring [10].
} 
For the discussion of the uniqueness of the left inverse as well as the analysis of the type of statistics of a sector the most important fact is the association of a "statistics parameter" $\lambda$ with each sector $\hat{\varrho}$ :

3.7. Theorem. If $\varrho \in \Delta_{t}$ is irreducible, $\varepsilon_{\varrho}$ as defined in Lemma 2.6 and $\phi$ a left inverse of $\varrho$ then

a) $\phi\left(\varepsilon_{0}\right)=\lambda I$.

b) The possible values of $\lambda$ are $0, \pm d^{-1}$ where $d$ is an integer.

c) The value of $\lambda$ associated with a sector by (3.16) is unique.

Proof. Statement a) follows from the fact that $\varepsilon_{\varrho}$ commutes with $\varrho^{2}(\mathfrak{A})$ (Eq. (2.13)):

$$
0=\phi\left(\varepsilon_{\varrho} \varrho^{2}(A)-\varrho^{2}(A) \varepsilon_{\varrho}\right)=\phi\left(\varepsilon_{\varrho}\right) \varrho(A)-\varrho(A) \phi\left(\varepsilon_{\varrho}\right), \quad A \in \mathfrak{A} .
$$

If $\varrho$ is irreducible we have (3.16). We shall postpone the proof of b) until section $\mathrm{V}$ because the relevant computations are facilitated by the algorithm developed in the next section. Statement c) follows from b) and Lemma 3.4. Suppose there exist two left inverses $\phi_{1}, \phi_{2}$ of $\varrho$ leading to values $\lambda_{1}, \lambda_{2}$ by (3.16). Then any convex combination of $\lambda_{1}, \lambda_{2}$ should occur also. But this is forbidden by $b$ ) because the values of $\lambda$ are restricted to a discrete set. Changing from $\varrho$ to an equivalent $\varrho^{\prime}=\sigma_{W} \varrho$ will not affect the value of $\lambda$ either: the change in $\varepsilon_{o}$ is given by (2.12), a left inverse of $\varrho^{\prime}$ is given by $\phi^{\prime}=\phi \sigma_{W^{*}}$. One checks that $\phi^{\prime}\left(\varepsilon_{Q^{\prime}}\right)=W \phi\left(\varepsilon_{\varrho}\right) W^{*}=\lambda I$. Thus $\lambda$ depends only on the sector $\hat{\varrho}$.

3.8. Lemma. Let $\varrho \in \Delta_{t}$ be irreducible, $\phi_{0}$ a left inverse of $\varrho$ obtained according to Lemma 3.3 as a limit point of the charge transfer chain $\sigma_{U_{k}}$ and $\phi$ any left inverse of $\varrho$. Then

$$
\begin{gathered}
\left\|\phi\left(A^{*} A\right)\right\| \geqq \lambda^{2}\left\|A^{*} A\right\| \\
\phi\left(A^{*} A\right) \geqq \lambda^{2} \phi_{0}\left(A^{*} A\right)
\end{gathered}
$$

where $\lambda$ is defined in Theorem 3.7 .

Proof. As $\phi$ is norm continuous it suffices to prove this result for $A$ having support in some finite region $\mathcal{O}$. Then for sufficiently large $k$ we have

so

$$
U_{k}^{*} A=\varrho(A) U_{k}^{*}=\varrho(A) \varepsilon_{\varrho} \varrho\left(U_{k}^{*}\right)
$$

and by (3.15) we have

$$
\phi\left(U_{k}^{*} A\right)=\lambda A U_{k}^{*}
$$

$$
\phi\left(A^{*} A\right) \geqq \phi\left(U_{k}^{*} A\right)^{*} \phi\left(U_{k}^{*} A\right)=\lambda^{2} U_{k} A^{*} A U_{k}^{*} .
$$

This gives (3.17) and taking the limit for a convergent subnet of the $\sigma_{U_{k}}$ we get (3.18). 
We now have all the machinery to demonstrate

3.9. Theorem. a) If $\varrho \in \Delta_{t}$ is irreducible and has finite statistics $(\lambda \neq 0)$ then @ has a unique left inverse $\phi$.

b) The sequence $\sigma_{U_{k}}$ converges to $\phi$ in $\mathscr{M}$.

c) There is a representation $\pi$, unique to within equivalence such that $\pi \circ \varrho$ contains the vacuum representation and the corresponding subspace contains a cyclic vector for $\pi$. This representation is irreducible and locally normal.

Proof. According to Lemma 3.4 we can apply the Krein-Milman Theorem to the set of all left inverses of $\varrho$. Let $\phi$ be an extremal left inverse and $\phi_{0}$ be one obtained from a convergent subnet of the sequence $\sigma_{U_{k}}$. If $\lambda \neq 0$ we can write

where

$$
\phi=\left(1-\eta^{2}\right) \phi_{1}+\eta^{2} \phi_{0} ; \quad 0<\eta^{2}<\lambda^{2}
$$

$$
\phi_{1}=\frac{\phi-\eta^{2} \phi_{0}}{1-\eta^{2}}
$$

is still a left inverse since, according to (3.18), it is still a positive map. The decomposition (3.19) contradicts the assumption that $\phi$ is extremal, unless $\phi=\phi_{0}$. Hence the set of left inverses has only one extremal point and therefore only one element. This proves part a) of the theorem. Part b) is, of course, an immediate consequence of the uniqueness of $\phi$. To prove c) let $\mathscr{H}$ be the representation space of $\pi$ and $\mathscr{H}_{1}$ a subspace such that the restriction of $\pi \circ \varrho$ to $\mathscr{H}_{1}$ is equivalent to $\pi_{0}$. Then we have

$$
\pi \circ \varrho(A) V=V A, \quad A \in \mathfrak{A}
$$

where $V$ is an isometric mapping of $\mathscr{H}_{0}$ into $\mathscr{H}$ with range $\mathscr{H}_{1}$. Let $E \in \pi(\mathfrak{2})^{\prime}$ be a non-zero projection then $V^{*} E V \in \mathfrak{U} \mathfrak{I}^{\prime}=\{\lambda I\}$. By assumption there is a unit vector $\xi=V \chi, \chi \in \mathscr{H}_{0}$, cyclic for $\pi(\mathfrak{Q})$ and hence separating for $\pi(\mathfrak{H})^{\prime}$. Thus $V^{*} E V=(\xi, E \xi) I \neq 0$ and defining $\phi$ by

$$
(\xi, E \xi) \phi(A)=V^{*} E \pi(A) E V, \quad A \in \mathfrak{A}
$$

$\phi$ is evidently a positive linear map with $\phi(I)=I$ and satisfies (3.5) and (3.6). Hence $\phi$ is the unique left inverse of $\varrho$ and is in particular independent of $E$. Thus setting $E=I$ and comparing with (3.21) we get

$$
(\xi, E \xi) V^{*} \pi(A) V=V^{*} E \pi(A) E V=V^{*} \pi(A) E V, \quad A \in \mathfrak{A} .
$$

So by taking expectation values in the vector $\chi$,

$$
(\xi, E \xi)(\xi, \pi(A) \xi)=(\xi, \pi(A) E \xi), \quad A \in \mathfrak{A} .
$$

Since $\xi$ is cyclic for $\pi(\mathfrak{Q})$ by assumption, $E \xi=(\xi, E \xi) \xi$ so $E=I$ and $\pi$ is irreducible. Furthermore the pure state $\omega_{0} \circ \phi$ is a vector state of $\pi$ (given 
by the vector $V \Omega$ ) so $\pi$ is unitarily equivalent to the representation $\pi_{\phi}$ of Lemma 3.5. $\omega_{0} \in \phi$ is locally normal because it is the limit of a sequence $\omega_{0} \circ \sigma_{U_{k}}$ of locally normal states. Hence $\pi$ itself is locally normal completing the proof.

\section{Intertwiners and Permutation Symmetry}

The purpose of this section is to develop a convenient notation and algorithm so that the generalization of Lemma 2.6 to an arbitrary number of factors becomes easily understandable.

Consider two localized morphisms $\varrho, \varrho^{\prime}$. If the corresponding representations of $\mathfrak{A}$ are not disjoint there exists an intertwining operator $R \in \mathscr{B}\left(\mathscr{H}_{0}\right)$ i.e.

$$
\varrho^{\prime}(A) R=R \varrho(A), \quad A \in \mathfrak{A} .
$$

As a shorthand expression for the statement that the triple $\varrho, \varrho^{\prime}$ and $R$ satisfy the relation (4.1) we write the symbol

$$
\boldsymbol{R}=\left(\varrho^{\prime}|R| \varrho\right)
$$

and call $\boldsymbol{R}$ an intertwiner. The adjoint of (4.2) is obviously

$$
\boldsymbol{R}^{*}=\left(\varrho\left|R^{*}\right| \varrho^{\prime}\right) \text {. }
$$

The composition of two intertwiners is defined if the adjoining morphisms coincide:

$$
\left(\varrho^{\prime \prime}|S| \varrho^{\prime}\right) \circ\left(\varrho^{\prime}|R| \varrho\right)=\left(\varrho^{\prime \prime}|S R| \varrho\right) .
$$

Consider the localization properties of intertwiners. In (4.2) let $\mathcal{O}_{1}$ be a support region of $\varrho$ and $\mathcal{O}_{2}$ one for $\varrho^{\prime}$. We call $\mathcal{O}_{2}$ a left support and $\mathcal{O}_{1}$ a right support of $\boldsymbol{R}$. The operator $R$ is bilocal in the sense that $R \in\left\{\mathfrak{A}\left(\mathcal{O}_{1}^{\prime}\right) \cap \mathfrak{A}\left(\mathcal{O}_{2}^{\prime}\right)\right\}^{\prime}$. In particular, if $\mathcal{O}$ is a double cone containing both $\mathcal{O}_{1}$ and $\mathcal{O}_{2}$ then $R \in \mathfrak{A}(\mathcal{O})$ by duality.

There is one more important operation on the set of intertwiners considered here. It arises from the multiplicative structure of the representations of interest. If we know intertwining operators from $\varrho_{i}$ to $\varrho_{i}^{\prime}$, $i=1,2$, then we can immediately write down an intertwining operator from $\varrho_{2} \varrho_{1}$ to $\varrho_{2}^{\prime} \varrho_{1}^{\prime}$. Explicitly if $\boldsymbol{R}_{i}=\left(\varrho_{i}^{\prime}\left|\boldsymbol{R}_{i}\right| \varrho_{i}\right), i=1,2$, we write

$$
\boldsymbol{R}_{2} \times \boldsymbol{R}_{1}=\left(\varrho_{2}^{\prime} \varrho_{1}^{\prime}\left|R_{2} \varrho_{2}\left(R_{1}\right)\right| \varrho_{2} \varrho_{1}\right) .
$$

Elementary calculation shows that this cross product is associative and one finds the formulae

$$
\begin{aligned}
(\boldsymbol{S} \times \boldsymbol{T})^{*} & =\boldsymbol{S}^{*} \times \boldsymbol{T}^{*}, \\
\left(\boldsymbol{R}_{2}^{\prime} \circ \boldsymbol{R}_{2}\right) \times\left(\boldsymbol{R}_{1}^{\prime} \circ \boldsymbol{R}_{1}\right) & =\left(\boldsymbol{R}_{2}^{\prime} \times \boldsymbol{R}_{1}^{\prime}\right) \circ\left(\boldsymbol{R}_{2} \times \boldsymbol{R}_{1}\right),
\end{aligned}
$$


the latter relation being valid when the left hand side is defined, i.e. when

$$
\boldsymbol{R}_{i}^{\prime}=\left(\varrho_{i}^{\prime \prime}\left|R_{i}^{\prime}\right| \varrho_{i}^{\prime}\right) ; \quad \boldsymbol{R}_{i}=\left(\varrho_{i}^{\prime}\left|R_{i}\right| \varrho_{i}\right) .
$$

We call two intertwiners causally disjoint if their right supports lie spacelike to each other and the same holds for their left supports.

4.1. Lemma. If $\boldsymbol{R}_{1}$ and $\boldsymbol{R}_{2}$ are causally disjoint then

$$
\boldsymbol{R}_{1} \times \boldsymbol{R}_{2}=\boldsymbol{R}_{2} \times \boldsymbol{R}_{1} .
$$

Proof. We have to show that under the stated conditions for the supports

$$
R_{1} \varrho_{1}\left(R_{2}\right)=R_{2} \varrho_{2}\left(R_{1}\right) .
$$

This relation is trivial if the four relevant supports are so situated that one can find two double cones $\hat{\mathcal{O}}_{1}$ and $\hat{\mathcal{O}}_{2}$ spacelike to each other and with $\hat{\mathcal{O}}_{i}$ containing both the right and the left support of $\boldsymbol{R}_{i}$. Let us then assume that (4.8) holds for a particular pair $\boldsymbol{R}_{1}, \boldsymbol{R}_{2}$ and let us shift for instance the right support of $\boldsymbol{R}_{2}$ (region $\mathcal{O}_{2}$ ) to $\mathcal{O}_{3}$ in such a way that the smallest double cone containing $\mathcal{O}_{2}$ and $\mathcal{O}_{3}$ is still spacelike to $\mathcal{O}_{1}$, the right support of $\boldsymbol{R}_{1}$. Thus we replace $\boldsymbol{R}_{2}$ by

$$
\boldsymbol{R}_{3}=\boldsymbol{R}_{2} \circ \boldsymbol{U} ; \quad \boldsymbol{U}=\left(\varrho_{2}|U| \varrho_{3}\right)
$$

where $U$ is a unitary localized spacelike to $\mathcal{O}_{1}$. Setting

we get from (4.7)

$$
\boldsymbol{I}_{\varrho} \equiv(\varrho|I| \varrho)
$$

$$
\begin{aligned}
& \boldsymbol{R}_{1} \times \boldsymbol{R}_{3}=\left(\boldsymbol{R}_{1} \times \boldsymbol{R}_{2}\right) \circ\left(I_{\varrho_{1}} \times \boldsymbol{U}\right) \\
& \boldsymbol{R}_{3} \times \boldsymbol{R}_{1}=\left(\boldsymbol{R}_{2} \times \boldsymbol{R}_{1}\right) \circ\left(U \times I_{\varrho_{1}}\right) .
\end{aligned}
$$

But the supports of $U$ and $\varrho_{1}$ are mutually spacelike so

$$
\boldsymbol{I}_{\varrho_{1}} \times \boldsymbol{U}=\boldsymbol{U} \times \boldsymbol{I}_{\varrho_{1}} .
$$

Thus the commutativity is preserved when we shift the right support of $\boldsymbol{R}_{2}$ as specified above. By a succession of small shifts of this nature we can bring this support from any one region spacelike to $\mathcal{O}_{1}$ to any other region spacelike to $\mathcal{O}_{1}$. The same procedure can then be applied to the right support of $\boldsymbol{R}_{\mathbf{1}}$. This establishes the lemma.

We can now describe the dependence of the cross product on the order of the factors in the general case. Given $n$ intertwiners $\boldsymbol{R}_{k}$ and a permutation $p=\left(\begin{array}{ccc}1 & \ldots \ldots . . & n \\ p(1) & \ldots & p(n)\end{array}\right)$ we use the shorthand notation

$$
\boldsymbol{R}(p)=\boldsymbol{R}_{p^{-1}(1)} \times \boldsymbol{R}_{p^{-1}(2)} \times \cdots \times \boldsymbol{R}_{p^{-1}(n)} .
$$


First we observe that for any $n$ morphisms $\varrho_{1}, \ldots, \varrho_{n}$ the equivalence class of the product does not depend on the order of factors. In fact there is a natural unitary intertwining operator between the representations $\varrho_{1} \ldots \varrho_{n}$ and $\varrho_{p(1)} \ldots \varrho_{p(n)}$. Its construction and properties are described in the following two theorems.

4.2. Theorem. Given $\varrho_{k} \in \Delta_{t} ; k=1,2, \ldots, n$ and $p \in P^{(n)}$. Choose $n$ morphisms $\varrho_{1}^{(0)}, \ldots, \varrho_{n}^{(0)}$ with mutually spacelike supports and equivalent to $\varrho_{1}, \ldots, \varrho_{n}$ respectively so that there exist unitary intertwiners $\left(\varrho_{k}^{(0)}\left|U_{k}\right| \varrho_{k}\right)$. Then set

$$
\begin{aligned}
\boldsymbol{U}^{*}(p) \circ \boldsymbol{U}(e)= & \boldsymbol{\varepsilon}_{p}\left(\varrho_{1}, \ldots, \varrho_{n}\right) \\
& =\left(\varrho_{p^{-1}(1)} \ldots \varrho_{p^{-1}(n)}\left|\varepsilon_{p}\left(\varrho_{1}, \ldots, \varrho_{n}\right)\right| \varrho_{1} \ldots \varrho_{n}\right)
\end{aligned}
$$

where the notation (4.10) has been used and e denotes the identity element of $P^{(n)}$. Then

a) $\boldsymbol{\varepsilon}_{p}$ is independent of the choice of the $\varrho_{k}^{(0)}$ and $U_{k}$ within the specified limitations.

b) $\varepsilon_{p}\left(\varrho_{1}, \ldots, \varrho_{n}\right)=I$ if the $\varrho_{k}$ have mutually spacelike supports.

c) $\boldsymbol{\varepsilon}_{q}\left(\varrho_{p^{-1}(1)}, \ldots, \varrho_{p^{-1}(n)}\right){ }^{\circ} \boldsymbol{\varepsilon}_{p}\left(\varrho_{1}, \ldots, \varrho_{n}\right)=\boldsymbol{\varepsilon}_{q p}\left(\varrho_{1}, \ldots, \varrho_{n}\right), p, q \in P^{(n)}$.

d) If $\tau_{m} \in P^{(n)}$ denotes the transposition of $m$ and $m+1, m<n$ $\boldsymbol{\varepsilon}_{\tau_{m}}\left(\varrho_{1}, \varrho_{2}, \ldots, \varrho_{n}\right)=\boldsymbol{I}_{\varrho_{1}} \times \cdots \times \boldsymbol{I}_{\varrho_{m-1}} \times \boldsymbol{\varepsilon}_{\tau}\left(\varrho_{m}, \varrho_{m+1}\right) \times \boldsymbol{I}_{\varrho_{n+1}} \times \cdots \times \boldsymbol{I}_{\varrho_{n}}$.

The operators $\varepsilon_{p}$ can be used to change the order in the cross product of any set of intertwiners:

4.3. Theorem. Given intertwiners $\boldsymbol{R}_{k}=\left(\varrho_{k}^{\prime}\left|R_{k}\right| \varrho_{k}\right), k=1, \ldots, n$ and $a$ permutation $p \in P^{(n)}$. Then

$$
\boldsymbol{R}(p) \circ \boldsymbol{\varepsilon}_{p}\left(\varrho_{1}, \ldots, \varrho_{n}\right)=\boldsymbol{\varepsilon}_{p}\left(\varrho_{1}^{\prime}, \ldots, \varrho_{n}^{\prime}\right) \circ \boldsymbol{R}(e)^{15} .
$$

Proof of Theorem 4.2. In (4.11) $U(e)$ intertwines from $\varrho_{1} \ldots \varrho_{n}$ to $\varrho_{1}^{(0)} \ldots \varrho_{n}^{(0)}$ and $U^{*}(p)$ from $\varrho_{p^{-1}(1)}^{(0)} \ldots \varrho_{p^{-1}(n)}^{(0)}$ to $\varrho_{p^{-1}(1)} \ldots \varrho_{p^{-1}(n)}$. The $\varrho_{k}^{(0)}$ having mutually spacelike supports all commute and thus the right argument of $\boldsymbol{U}^{*}(p)$ coincides with the left argument of $\boldsymbol{U}(e)$. Therefore the composition (4.11) is well defined and leads to an intertwiner as specified in (4.11). To see that $\varepsilon_{p}$ is independent of the choice of $\varrho_{k}^{(0)}, \boldsymbol{U}_{k}$ let us take $\tilde{\boldsymbol{U}}_{k}=\left(\tilde{\varrho}_{k}^{(0)}\left|\tilde{U}_{k}\right| \varrho_{k}\right)$ instead of $\boldsymbol{U}_{k}$, where the $\tilde{\varrho}_{k}^{(0)}$ again have mutually spacelike supports. Then $\boldsymbol{V}_{k}=\tilde{\boldsymbol{U}}_{k}{ }^{\circ} \boldsymbol{U}_{k}^{*}$ intertwines from $\varrho_{k}^{(0)}$ to $\tilde{\varrho}_{k}^{(0)}$. Hence the $\boldsymbol{V}_{k}$ are causally disjoint intertwiners and we have $\boldsymbol{V}(p)=\boldsymbol{V}(e)$ by Lemma 4.1. Using formula (4.7) this may be written

$$
\tilde{\boldsymbol{U}}(p) \circ \boldsymbol{U}^{*}(p)=\tilde{\boldsymbol{U}}(e) \circ \boldsymbol{U}^{*}(e)
$$

${ }^{15}$ For those familiar with category theory we remark that localized morphisms and intertwiners form a symmetric monoidal category in the language of Eilenberg and Kelly [11]. $\varepsilon$ is the natural transformation making the monoidal structure symmetric.

16 Commun. math. Phys., Vol. 23 
or

$$
\tilde{U}^{*}(p) \circ \tilde{\boldsymbol{U}}(e)=\boldsymbol{U}^{*}(p) \circ \boldsymbol{U}(e)
$$

which is the desired statement of independence. Part b) follows immediately from the independence because, if the $\varrho_{k}$ have mutually spacelike supports we may choose $U_{k}=\left(\varrho_{k}|I| \varrho_{k}\right)$. Part c) follows immediately from (4.11) and so does part d) when relation (4.7) is used.

Proof of Theorem 4.3. Let us choose unitary intertwiners $\left(\varrho_{k}^{(0)}\left|U_{k}\right| \varrho_{k}\right)$ and $\left(\varrho_{k}^{(0)^{\prime}}\left|U_{k}^{\prime}\right| \varrho_{k}^{\prime}\right)$ where the supports of the morphisms $\varrho_{k}^{(0)}$ as well as those of the $\varrho_{k}^{(0)^{\prime}}$ are mutually spacelike. Define

$$
\boldsymbol{S}_{\boldsymbol{k}}=\boldsymbol{U}_{\boldsymbol{k}}^{\prime} \circ \boldsymbol{R}_{\boldsymbol{k}} \circ \boldsymbol{U}_{k}^{*} .
$$

By construction the $S_{k}$ are all causally disjoint. Hence

$$
\boldsymbol{S}(p)=\boldsymbol{S}(e) .
$$

Insert (4.14) into (4.15), use (4.7) and rearrange to obtain Eq. (4.13).

Comments. Theorem 4.2 reduces to Lemma 2.6 in the special case $n=2, p=\tau$ (transposition) and $\varrho_{1}=\varrho_{2}$. The notation in section II is translated into the one of this section by the identification

$$
\varepsilon_{\tau}(\varrho, \varrho) \equiv \varepsilon_{\varrho} .
$$

For $n>2$ the case where all morphisms $\varrho_{i}$ are equal is also of special interest. We shall then write, in analogy with (4.16)

$$
\varepsilon_{p}(\varrho, \varrho, \ldots, \varrho)=\varepsilon_{\varrho}^{(n)}(p) .
$$

An immediate corollary of Theorems 4.2 and 4.3 and Lemma 2.7 is

4.4. Proposition. a) $p \rightarrow \varepsilon_{Q}^{(n)}(p)$ is a unitary representation of the permutation group $P^{(n)}$.

b) $\varepsilon_{\varrho}^{(n)}(p)$ commutes with $\varrho^{n}(\mathfrak{Q})$.

c) The operators $\varepsilon_{e}^{(n)}(p)$ are scalar multiples of the identity if and only if $\varrho$ is an automorphism.

d) If $\boldsymbol{W}=\left(\varrho^{\prime}|W| \varrho\right)$ is unitary and $\boldsymbol{W}^{\times n}$ denotes the $n$-fold $\times$-product of $\boldsymbol{W}$ with itself

$$
\varepsilon_{\ell^{\prime}}^{(n)}(p)=W^{\times n} \circ \varepsilon_{\varrho}^{(n)}(p) \circ\left(W^{\times n}\right)^{*} .
$$

Proposition $4.4 \mathrm{~d}$ generalizes Lemma $2.6 \mathrm{~b}$ and implies that the equivalence class of the unitary representation $\varepsilon_{\varrho}^{(n)}$ of $P^{(n)}$ depends only on $\hat{\varrho}$.

The significance of the operators $\varepsilon_{e}^{(n)}(p)$ has been described in the introduction. We can now verify in detail the remarks made there. Let $\varrho_{k} \in \Delta_{t}, k=1, \ldots, n$, belong to the same equivalence class and have mutually spacelike supports. Pick a reference morphism $\varrho$ in this class and intertwiners $\left(\varrho_{k}\left|U_{k}\right| \varrho\right)$. In the representation $\varrho$ the vector $U_{k}^{*} \Omega \in \mathscr{H}_{0}$ 
represents the state $\omega_{0} \circ \varrho_{k}$ :

$$
\left(U_{k}^{*} \Omega, \varrho(A) U_{k}^{*} \Omega\right)=\left(\Omega, \varrho_{k}(A) \Omega\right)=\omega_{0}\left(\varrho_{k}(A)\right) .
$$

Consider now the vector

$$
|p\rangle=U^{*}(p) \Omega=U_{p^{-1}(1)}^{*} \times \cdots \times U_{p^{-1}(n)}^{*} \Omega,
$$

where $p \in P^{(n)}$. This corresponds to a product of $n$ state vectors (with identical charge quantum numbers) as mentioned in the introduction. The order of factors is determined by $p$. This vector must be considered in the representation $\varrho^{n}$ where it represents the state $\omega_{0}{ }^{\circ} \varrho_{1} \varrho_{2} \ldots \varrho_{n}$ irrespective of which permutation $p$ was chosen, because the $\varrho_{k}$ commute. Proposition 4.4 tells us that the vectors $|p\rangle$ defined in (4.19) are transformed into each other by the permutation operators $\varepsilon_{e}^{(n)}(q)$ :

$$
\varepsilon_{\varrho}^{(n)}(q)|p\rangle=|q p\rangle .
$$

The permutation operators commute with all observables (Proposition 4.4) so the vectors $|p\rangle$ represent the same state for all $p \in P^{(n)}$. These properties of the permutation operators make them completely analogous to the place permutations of the wave functions of $n$ identical particles in wave mechanics.

\section{The Statistics of a Sector}

As described in the introduction, we call the statistics of the sector $\hat{\varrho}$ the collection of equivalence classes of the representations $\varepsilon_{e}^{(n)}$ of the permutation groups $P^{(n)}$ as $n$ varies. The main concern of this section is to classify the possible statistics. We note first that each irreducible component of $\varepsilon_{e}^{(n)}$ occurs with multiplicity $\operatorname{dim} \mathscr{H}_{0}$. In fact if $E \neq 0$ is the corresponding central projection then $E \in \varrho^{n}(\mathfrak{A})^{\prime} \subset \mathfrak{A}(\mathcal{O})$, where $\mathcal{O}$ is the support of $\varrho$, and Property B shows that $E$ projects onto a subspace with the same dimension as $\mathscr{H}_{0}$. Hence it suffices to determine which irreducible components occur, i.e. to determine the quasiequivalence class of $\varepsilon_{e}^{(n)}$. Irreducible representations of $P^{(n)}$ correspond to Young tableaux with $n$ squares and we consider our goal achieved when we describe the Young tableaux associated with $\varepsilon_{e}^{(n)}$ as $n$ varies.

The simplest and most important case is where $\varrho$ is irreducible and we recall from Theorem 3.7a that, if $\phi$ is a left inverse of an irreducible $\varrho, \phi\left(\varepsilon_{\varrho}\right)$ is a multiple of the identity.

5.1. Lemma. Let $\phi$ be a left inverse for $\varrho \in \Delta_{t}$ with $\phi\left(\varepsilon_{\varrho}\right)=\lambda I$ then if $p \in P^{(n)}$

$$
\begin{array}{ll}
\phi\left(\varepsilon_{\varrho}^{(n)}(p)\right)=\varepsilon_{\varrho}^{(n-1)}\left(p^{\prime}\right) & p(1)=1 \\
\phi\left(\varepsilon_{\varrho}^{(n)}(p)\right)=\lambda \varepsilon_{\varrho}^{(n-1)}\left(p^{\prime}\right) & p(1) \neq 1
\end{array}
$$


where $p^{\prime} \in P^{(n-1)}$ is the permutation obtained from $p$ by deleting 1 from its cycle in the decomposition of $p$ into disjoint cycles and then writing $t$ for $t+1, t=1,2, \ldots, n-1$.

Proof. If $p(1)=1, \varepsilon_{e}^{(n)}(p)=\varrho\left(\varepsilon_{e}^{(n-1)}\left(p^{\prime}\right)\right)$ as follows, for example, from Theorem 4.2d. If $p(1) \neq 1$, write $p=p_{1} p_{2} p_{3}$ where $p_{2}=(12), p_{1}=(2 p(1))$ and the symbol (st) denotes the transposition of $s$ and $t$. This defines $p_{3}$ and one checks that $p_{3}(1)=1$. By Theorem 4.2d and Eqs. (3.5) and (3.6) we get

$$
\phi\left(\varepsilon_{\varrho}^{(n)}\right)=\varepsilon_{\varrho}^{(n-1)}\left(p_{1}^{\prime}\right) \phi\left(\varepsilon_{\varrho}\right) \varepsilon_{\varrho}^{(n-1)}\left(p_{3}^{\prime}\right)=\lambda \varepsilon_{\varrho}^{(n-1)}\left(p_{1}^{\prime} p_{3}^{\prime}\right) .
$$

The proof is completed by checking that $p_{1}^{\prime} p_{3}^{\prime}=p^{\prime}$.

5.2. Proposition. Let $\phi$ be a left inverse for $\varrho \in \Delta_{t}$ with $\phi\left(\varepsilon_{\varrho}\right)=\lambda I$, then

$$
\phi^{n-1}\left(\varepsilon_{\varrho}^{(n)}(p)\right)=\omega_{\lambda}^{n}(p) I, \quad p \in P^{(n)}
$$

where $\omega_{\lambda}^{n}$ is the trace state on $P^{(n)}$ multiplicative on disjoint cycles and taking the value $\lambda^{k-1}$ on a $k$-cycle. The cyclic representation of $P^{(n)}$ generated by $\omega_{\lambda}^{n}$ is quasiequivalent to $\varepsilon_{\varrho}^{(n)}$.

Proof. Eq. (5.1) follows by using Lemma 5.1 repeatedly. Since $\phi$ is a positive map with $\phi(I)=I, \omega_{\lambda}^{n}$ is a state. Let $E$ be a central projection in the group algebra of $P^{(n)}$ then $\varepsilon_{\varrho}^{(n)}(E)=0$ implies $\omega_{\lambda}^{n}(E)=0$ trivially. However if $\lambda \neq 0$ the converse follows because $\phi$ is then faithful by (3.17). On the other hand if $\lambda=0, \omega_{\lambda}^{n}$ is the state that generates the left regular representation of $P^{(n)}$ so $\omega_{\lambda}^{n}(E)=0$ implies $E=0$. Hence in either case the cyclic representation of $P^{(n)}$ generated by $\omega_{\lambda}^{n}$ is quasiequivalent to $\varepsilon_{o}^{(n)}$.

This result shows that the statistics of a sector is determined by the value of $\lambda$. The problems that remain are of a group theoretical nature: we must determine the possible values of $\lambda$ and the Young tableaux associated with the state $\omega_{\lambda}^{n}$.

5.3. Lemma. Let $E_{s}^{n}$ and $E_{a}^{n}$ denote the symmetric and antisymmetric projections in the group algebra of $P^{(n)}$ then

$$
\begin{aligned}
& \omega_{\lambda}^{n}\left(E_{s}^{n}\right)=\frac{1}{n !}(1+\lambda)(1+2 \lambda) \ldots(1+(n-1) \lambda), \\
& \omega_{\lambda}^{n}\left(E_{a}^{n}\right)=\frac{1}{n !}(1-\lambda)(1-2 \lambda) \ldots(1-(n-1) \lambda) .
\end{aligned}
$$

The possible values of $\lambda$ are $0, \pm d^{-1}$ where $d$ is an integer.

Proof. Using the same way of passing from an element of $P^{(n)}$ to one of $P^{(n-1)}$ as was used in Lemma 5.1 we deduce that

$$
\omega_{\lambda}^{n}\left(E_{s}^{n}\right)=\frac{1}{n} \omega_{\lambda}^{n-1}\left(E_{s}^{n-1}\right)+\frac{n-1}{n} \lambda \omega_{\lambda}^{n-1}\left(E_{s}^{n-1}\right) .
$$


Furthermore noting that if an element is removed from a cycle of length $>1$ we change the sign of the permutation, we see that

$$
\omega_{\lambda}^{n}\left(E_{a}^{n}\right)=\frac{1}{n} \omega_{\lambda}^{n-1}\left(E_{a}^{n-1}\right)-\frac{n-1}{n} \lambda \omega_{\lambda}^{n-1}\left(E_{a}^{n-1}\right) .
$$

Induction on (5.4) and (5.5) leads to (5.2) and (5.3). As $\omega_{\lambda}^{n}$ is a state on $P^{(n)}, \omega_{\lambda}^{n}\left(E_{s}^{n}\right) \geqq 0$ and $\omega_{\lambda}^{n}\left(E_{a}^{n}\right) \geqq 0$ for all $n$ and the restriction on the values of $\lambda$ follows from (5.2) and (5.3).

This lemma completes the proof of Theorem $3.7 \mathrm{~b}$ and fills the gap in section III in the proof that an irreducible morphism $\varrho \in \Delta_{t}$ with $\lambda \neq 0$ has a unique left inverse. The next lemma shows that positivity implies no further restrictions on the values of $\lambda$.

5.4. Lemma. Let $\mathscr{H}$ be a Hilbert space of finite dimension d. Consider the natural representation $\pi$ of $P^{(n)}$ on $\mathscr{H} \otimes \mathscr{H} \otimes \cdots \otimes \mathscr{H}$ which acts by permuting factors and the representation $\pi^{\prime}$ defined by $\pi^{\prime}(p)=\operatorname{sign}(p) \pi(p)$ then

$$
\begin{aligned}
d^{-n} \operatorname{Tr}(\pi(p)) & =\omega_{d^{-1}}^{n}(p), \quad p \in P^{(n)}, \\
d^{-n} \operatorname{Tr}\left(\pi^{\prime}(p)\right) & =\omega_{-d^{-1}}^{n}(p), \quad p \in P^{(n)} .
\end{aligned}
$$

Proof. $\operatorname{Tr}(\pi(p))$ is a class function. Consider the decomposition of $p$ into disjoint cycles, $p=c_{1} c_{2} \ldots c_{m}$ say, then

$$
d^{-n} \operatorname{Tr}(\pi(p))=\prod_{i=1}^{m} d^{-n} \operatorname{Tr}\left(\pi\left(c_{i}\right)\right)
$$

However if $c$ is a $k$-cycle, which without loss of generality we may suppose to be $(12 \ldots k)$, then

$$
\operatorname{Tr}(\pi(c))=\sum_{\substack{i_{1}, \ldots, i_{k} \\ i_{k+1}, \ldots, i_{n}}}\left(\Phi_{i_{1}} \otimes \cdots \otimes \Phi_{i_{k}}, \Phi_{i_{k}} \otimes \Phi_{i_{1}} \otimes \cdots \otimes \Phi_{i_{k-1}}\right)
$$

where $\Phi_{i}, i=1,2, \ldots d$ runs over an orthonormal basis for $\mathscr{H}$. Thus $\operatorname{Tr}(\pi(c))=d^{n-k} d$ as required. This proves (5.6) and (5.7) follows similarly replacing $\pi$ by $\pi^{\prime}$.

5.5. Theorem. If $\phi$ is a left inverse for $\varrho \in \Delta_{t}$ with $\phi\left(\varepsilon_{\varrho}\right)=\lambda I$ the Young tableaux associated with the representations $\varepsilon_{e}^{(n)}$ of $P^{(n)}, n \geqq 1$ are all Young tableaux

a) whose columns have length $\leqq d$, if $\lambda=d^{-1}$ (para-Bose statistics of order d),

b) whose rows have length $\leqq d$, if $\lambda=-d^{-1}$ (para-Fermi statistics of order d),

c) without restriction, if $\lambda=0$ (infinite statistics). 
Proof. If $\lambda=0, \omega_{\lambda}^{n}$ is the natural state generating the left regular representation of $P^{(n)}$ so all Young tableaux occur. If $\lambda=d^{-1}, \varepsilon_{e}^{(n)}$ is quasiequivalent to the representation $\pi$ of Lemma 5.4. For $\pi$ we may proceed by projecting out parts of the natural basis vectors transforming irreducibly under $P^{(n)}$ using projections associated in the usual way (see e.g. [12]) with the corresponding Young tableaux. The symmetrizations involved pose no problem however antisymmetrizing $\Phi_{i_{1}} \otimes \cdots \otimes \Phi_{i_{n}}$ with respect to a subset of its indices leads to a nonzero result only if the corresponding components are distinct. This yields a) and b) follows by interchanging rows and columns.

We see that under our assumptions a sector has either para-Bose, para-Fermi or infinite statistics. As indicated in the introduction there are no known examples of infinite statistics and arguments why they should not occur will be given in the sequel.

\section{Reducible Representations}

Further insight into the structure of localized morphisms can be gained by trying to analyze the statistics of reducible representations. It is important to realize that if a reducible $\varrho$ has a left inverse $\phi$ with $\phi\left(\varepsilon_{\varrho}\right)=\lambda I$ then the analysis of section V still applies and the statistics of $\varrho$ is characterized by Theorem 5.5. It will turn out that reducible $\varrho$ do not have this property in general and their statistics is then neither para-Bose, para-Fermi nor infinite but rather a mixture of para-Bose and para-Fermi statistics.

On physical grounds one would expect that any reducible representation satisfying criterion 1.1 is the direct sum of irreducible representations. We show first that this is the case at least if we exclude infinite statistics.

6.1. Lemma. Let $\phi$ be a left inverse of $\varrho \in \Delta_{t}$ and $F$ a spectral projection of the self-adjoint operator $\Lambda \equiv \phi\left(\varepsilon_{e}\right)$ corresponding to $\{t \in \boldsymbol{R}:|t| \geqq \delta\}$, $\delta>0$. Then there are at most a finite number $m$ of mutually orthogonal projections $E_{i} \in \varrho(\mathfrak{Q})^{\prime}$ with $E_{i} \leqq F$.

Proof. Let $E \in \varrho(\mathfrak{Q})^{\prime}$ be a projection with $0<E \leqq F$ and let $\boldsymbol{U}=\left(\varrho^{\prime}|U| \varrho\right)$ be a unitary intertwiner where $\varrho$ and $\varrho^{\prime}$ have spacelike separated supports. Then $\varrho^{\prime}(E)=E$ and from (2.11) we get

$$
U^{-1} E=\varrho(E) U^{-1}=\varrho(E) \varepsilon_{\varrho} \varrho(U)^{-1} .
$$

Using (3.5), (3.6) and (3.15) we deduce

$$
\phi(E) \geqq \phi\left(U^{-1} E\right)^{*} \phi\left(U^{-1} E\right)=U \Lambda E \Lambda U^{-1} .
$$

Now $\left\|U \Lambda E \Lambda U^{-1}\right\|=\|\Lambda E \Lambda\|=\|\Lambda E\|^{2} \geqq \delta^{2}$ since $E \leqq F$. 
But $E \in \varrho(\mathfrak{R})^{\prime}$ implies $\phi(E) \in \mathfrak{A}^{\prime}$ so $\phi(E)$ is a multiple of the identity and hence from (6.1)

$$
\phi(E) \geqq \delta^{2} I .
$$

Now $I \geqq \phi(F) \geqq \sum_{i=1}^{m} \phi\left(E_{i}\right)$ so using (6.2) for each $E_{i}$ we deduce $m \leqq \delta^{-2}$ completing the proof.

6.2. Corollary. a) $\Lambda$ has a discrete spectrum.

b) $\varrho(\mathfrak{A})_{F}^{\prime}$ is a finite discrete von Neumann algebra.

c) If $\varrho(\mathfrak{A})^{\prime}$ is a continuous von Neumann algebra, $\phi\left(\varepsilon_{\varrho}\right)=0$ and $\varrho$ has infinite statistics.

From this corollary we see that insofar as we restrict ourselves to finite statistics, all representations are the direct sum of irreducible representations.

To classify the statistics of reducible representations we need the concept of a standard left inverse. A left inverse $\phi$ of a $\varrho \in \Delta_{t}$ will be called standard if $\phi\left(\varepsilon_{e}\right)^{2}$ is a multiple of the identity. It will be noted that the left inverses used in Theorem 5.5 and in the results leading up to that theorem are standard.

6.3. Proposition. Every $\varrho \in \Delta_{t}$ has a standard left inverse. If $\varrho$ has a subrepresentation with infinite statistics then $\varrho$ has infinite statistics.

Proof. Let $\boldsymbol{W}_{i}=\left(\varrho\left|W_{i}\right| \varrho_{i}\right)$ be isometric intertwiners with

$$
\sum_{i=1}^{m} \boldsymbol{W}_{i} \circ \boldsymbol{W}_{i}^{*}=\boldsymbol{I}_{\varrho} \text { and } \boldsymbol{W}_{i}^{*} \circ \boldsymbol{W}_{j}=\delta_{i j} \boldsymbol{I}_{\varrho_{i}} .
$$

Let $\phi_{i}$ be a left inverse for $\varrho_{i}, i=1,2, \ldots, m$ and set

$$
\phi(A)=\sum_{i=1}^{m} a_{i} \phi_{i}\left(W_{i}^{*} A W_{i}\right), \quad A \in \mathfrak{A}
$$

where $a_{i} \geqq 0$ and $\sum_{i=1}^{m} a_{i}=1$. Then $\phi$ is clearly a left inverse for $\varrho$. Further by Theorem 4.3,

$$
\boldsymbol{\varepsilon}_{\varrho} \circ \boldsymbol{W}_{\boldsymbol{i}} \times \boldsymbol{W}_{j}=\boldsymbol{W}_{j} \times \boldsymbol{W}_{\boldsymbol{i}} \circ \boldsymbol{\varepsilon}_{\tau}\left(\varrho_{i}, \varrho_{j}\right) .
$$

Hence $W_{i}^{*} \varepsilon_{\varrho} W_{i} \varrho_{i}\left(W_{j}\right)=\delta_{i j} \varrho_{i}\left(W_{i}\right) \varepsilon_{Q_{i}}$ and thus $\phi_{i}\left(W_{i}^{*} \varepsilon_{\varrho} W_{i}\right) W_{j}=\delta_{i j} W_{i} \phi_{i}\left(\varepsilon_{\varrho_{i}}\right)$. So $\phi_{i}\left(W_{i}^{*} \varepsilon_{\varrho} W_{i}\right)=\sum_{j} \phi_{i}\left(W_{i}^{*} \varepsilon_{\varrho} W_{i}\right) W_{j} W_{j}^{*}=W_{i} \phi_{i}\left(\varepsilon_{\varrho_{i}}\right) W_{i}^{*}$. Then by (6.3)

$$
\phi\left(\varepsilon_{\varrho}\right)=\sum_{i=1}^{m} a_{i} W_{i} \phi_{i}\left(\varepsilon_{\varrho_{i}}\right) W_{i}^{*} .
$$

Suppose that $\varrho_{1}$ corresponds to a subrepresentation of $\varrho$ with infinite statistics then, by definition, we may take $\phi_{1}$ with $\phi_{1}\left(\varepsilon_{\varrho_{1}}\right)=0$. Setting 
$a_{1}=1, a_{i}=0, i \neq 1$ we deduce that $\phi\left(\varepsilon_{\varrho}\right)=0$ so $\varrho$ itself has infinite statistics. Suppose now that $\varrho_{i}, i=1,2, \ldots, m$ are irreducible with finite statistics, then, putting $E_{i}=W_{i} W_{i}^{*}$,

$$
\phi_{i}\left(\varepsilon_{Q_{i}}\right)=\lambda_{i} I \text { and } \phi\left(\varepsilon_{\varrho}\right)=\sum_{i=1}^{m} a_{i} \lambda_{i} E_{i} .
$$

Setting $a_{i}=d_{i} d^{-1}$ where $d_{i}=\left|\lambda_{i}\right|^{-1}$ and $d=\sum_{i=1}^{m} d_{i}$ we get

where

$$
\phi\left(\varepsilon_{\varrho}\right)=d^{-1}\left(E_{b}-E_{f}\right)
$$

$$
\begin{gathered}
E_{b}=\sum_{\lambda_{i}>0} E_{i} \text { and } E_{f}=\sum_{\lambda_{i}<0} E_{i} \\
\phi\left(\varepsilon_{Q}\right)^{2}=d^{-2}\left(E_{b}+E_{f}\right)=d^{-2} I .
\end{gathered}
$$

Thus $\phi$ is standard. It only remains to consider the case where $\varrho$ is an infinite direct sum of irreducibles $\varrho_{i}$ with finite statistics. Eq. (6.3) can still be used to define a left inverse, $\phi^{(m)}$ say, for $\varrho$ and (6.6) is still valid so we may arrange that $\phi^{(m)}\left(\varepsilon_{\rho}\right)$ converges weakly to zero. By Lemma 3.4 there is a limit point $\phi \in \mathscr{M}$ of the sequence $\left\{\phi^{(m)}\right\}$ and $\phi$ will be a left inverse for $\varrho$ with $\phi\left(\varepsilon_{\varrho}\right)=0$. Thus $\phi$ is a standard left inverse for $\varrho$ and $\varrho$ has infinite statistics.

The form of Eq. (6.7) already indicates that the only reason why the statistics of a reducible representation need not fall into one of the categories in Theorem 5.5 is that it may be a mixture of para-Bose and para-Fermi statistics. Before stating the general form for the statistics we collect together a few results on standard left inverses.

6.4. Lemma. If $\phi$ is a standard left inverse for $\varrho \in \Delta_{t}$ and $\phi\left(\varepsilon_{\varrho}\right) \neq 0$ then $\phi$ is faithful. In fact

$$
\left\|\phi\left(B^{*} B\right)\right\| \geqq\left\|\phi\left(\varepsilon_{\varrho}\right)^{2}\right\|\left\|B^{*} B\right\| .
$$

Proof. It suffices to prove (6.10) for $B \in \mathfrak{A}(\mathcal{O})$. Pick a unitary intertwiner $\boldsymbol{U}=\left(\varrho^{\prime}|U| \varrho\right)$ where $\varrho^{\prime}$ has support spacelike to $\mathcal{O}$ and to that of $\varrho$. Then $\varrho(B)=U^{-1} B U$ and $\varepsilon_{\varrho}=U^{-1} \varrho(U)$. Thus by (3.15), (3.5) and (3.6)

$$
\phi\left(B^{*} B\right) \geqq \phi\left(U^{-1} B\right)^{*} \phi\left(U^{-1} B\right)=U \phi\left(\varepsilon_{\varrho}\right) B^{*} B \phi\left(\varepsilon_{\varrho}\right) U^{-1} .
$$

Since $\left\|\phi\left(\varepsilon_{\varrho}\right)\right\|^{-1} \phi\left(\varepsilon_{\varrho}\right)$ is unitary the result now follows. then

6.5. Proposition. If $\phi$ is a standard left inverse for $\varrho \in \Delta_{t}$ and $\phi\left(\varepsilon_{\varrho}\right) \neq 0$

$$
\phi\left(\varepsilon_{\varrho}\right)=d^{-1}\left(E_{b}-E_{f}\right)
$$

where $E_{b}$ and $E_{f}$ are the central projections in $\varrho(\mathfrak{A})^{\prime}$ containing the projections onto the para-Bose and para-Fermi components of @ respectively. Further $d \phi\left(E_{b}\right)=b I$ and $d \phi\left(E_{f}\right)=f I$ where $b$ and $f$ are integers independent of the choice of $\phi$ and satisfying $d=b+f$. 
Proof. Since $\phi$ is standard we may use (6.11) to define $E_{b}$ and $E_{f}$. Let $E \in \varrho(\mathfrak{R})^{\prime}$ be a projection with $0<E \leqq E_{b}$, then by Lemma 2.5 there is an isometric intertwiner $\boldsymbol{W}=\left(\varrho|W| \varrho^{\prime}\right)$ with $W W^{*}=E$. Now $\phi(E)$ is a multiple of the identity and by Lemma $6.4, \phi(E) \neq 0$ so setting

$$
\phi^{\prime}(A)=\phi(E)^{-1} \phi\left(W A W^{*}\right)
$$

$\phi^{\prime}$ is a left inverse for $\varrho^{\prime}$. Further by Theorem 4.3

hence

$$
\boldsymbol{\varepsilon}_{\varrho^{\prime}}=\left(\boldsymbol{W}^{*} \times \boldsymbol{W}^{*}\right) \circ \boldsymbol{\varepsilon}_{\varrho} \circ(\boldsymbol{W} \times \boldsymbol{W}) \text { and } \varepsilon_{\varrho} E=\varrho(E) \varepsilon_{\varrho},
$$

$$
\phi^{\prime}\left(\varepsilon_{\varrho^{\prime}}\right)=\phi(E)^{-1} W^{*} \phi\left(\varepsilon_{\varrho}\right) W .
$$

Since $E \leqq E_{b}$, (6.13) gives $\phi^{\prime}\left(\varepsilon_{\varrho^{\prime}}\right)=d^{-1} \phi(E)^{-1}$. Thus $\phi^{\prime}$ is standard and $\varrho^{\prime}$ has para-Bose statistics of order $d \phi(E)$. Similarly if $0<E \leqq E_{f}, \varrho^{\prime}$ would have para-Fermi statistics of order $d \phi(E)$. Since the statistics is a unitary invariant and by Theorem 5.5 the para-Bose or para-Fermi orders are integers the remaining assertions follow.

The integers $b$ and $f$ defined above are thus unitary invariants; we shall denote them by $b(\varrho)$ and $f(\varrho)$ and call them the para-Bose order and para-Fermi order of $\varrho$ respectively. Their sum $d(\varrho)=b(\varrho)+f(\varrho)$ will be called the statistical dimension of $\varrho$. The condition $\phi\left(\varepsilon_{\varrho}\right) \neq 0$ appearing in Lemma 6.4 and Proposition 6.5 is just the condition that $\varrho$ shall have finite statistics. If $\varrho$ has infinite statistics we write $d(\varrho)=\infty$ conventionally, leaving $b(\varrho)$ and $f(\varrho)$ undefined.

6.6. Proposition. If $\phi$ is a standard left inverse for a $\varrho \in \Delta_{t}$ with finite statistics, then

$$
\phi(A)=\operatorname{Tr}_{\varrho}(A) I, \quad A \in \varrho(\mathfrak{U})^{\prime}
$$

where $\operatorname{Tr}_{\varrho}$ is a faithful trace state. Further if $E \in \varrho(\mathfrak{Q})^{\prime}$ is a non-zero projection, then

$$
\operatorname{Tr}_{\varrho}(E)=\frac{d(E)}{d(\varrho)}
$$

where $d(E)$ is the statistical dimension of the subrepresentation $\pi_{0} \circ \varrho \mid E \mathscr{H}_{0}$.

Proof. With the notation of Proposition 6.5, define a left inverse by (6.12) and then by (6.13)

$$
\phi^{\prime}\left(\varepsilon_{\varrho^{\prime}}\right)^{2} \phi(E)^{2}=W^{*} \phi\left(\varepsilon_{\varrho}\right) W W^{*} \phi\left(\varepsilon_{\varrho}\right) W=W^{*} \phi\left(\varepsilon_{\varrho}\right)^{2} W=\phi\left(\varepsilon_{\varrho}\right)^{2} .
$$

Here the second equality follows because (6.11) implies that $\phi\left(\varepsilon_{e}\right)$ is in the centre of $\varrho(\mathfrak{U})^{\prime}$. Thus $\phi^{\prime}$ is a standard left inverse for $\varrho^{\prime}$ and $\phi^{\prime}\left(\varepsilon_{Q^{\prime}}\right)^{2}=d(E)^{-2}$ giving (6.15). The statistical dimension is a unitary invariant so that $\phi(E)=\phi\left(U E U^{-1}\right)$ for every $U \in \varrho(\mathfrak{M})^{\prime}$. This suffices to establish that $\operatorname{Tr}_{\varrho}$ is a trace state on $\varrho(\mathfrak{A})^{\prime}$ and faithfulness follows from (6.15) or Lemma 6.4. 
From the explicit construction of a standard left inverse in Proposition 6.3 , it follows that $b$ and $f$ are additive on direct sums,

$$
b(\varrho)=\sum_{i=1}^{n} b\left(\varrho_{i}\right) \text { and } f(\varrho)=\sum_{i=1}^{n} f\left(\varrho_{i}\right) \text { if } \varrho=\bigoplus_{i=1}^{n} \varrho_{i} .
$$

We investigate next the behaviour of $b$ and $f$ on taking products of localized morphisms. If we let $\phi\left(\varepsilon_{\varrho}\right)=\left(\varrho\left|\phi\left(\varepsilon_{\varrho}\right)\right| \varrho\right)$ we have

6.7. Lemma. Let $\phi_{1}, \phi_{2}$ be left inverses for $\varrho_{1}, \varrho_{2} \in \Delta_{t}$ respectively then $\phi_{2} \phi_{1}$ is a left inverse for $\varrho_{1} \varrho_{2}$ and

$$
\phi_{2} \phi_{1}\left(\varepsilon_{\varrho_{1} Q_{2}}\right)=\phi_{1}\left(\varepsilon_{\varrho_{1}}\right) \times \phi_{2}\left(\varepsilon_{\varrho_{2}}\right) .
$$

Proof. Pick unitary intertwiners $\boldsymbol{U}_{1}=\left(\varrho_{1}^{\prime}\left|U_{1}\right| \varrho_{1}\right)$ and $\boldsymbol{U}_{2}=\left(\varrho_{2}^{\prime}\left|U_{2}\right| \varrho_{2}\right)$ where the support of $\varrho_{1}^{\prime}$ is spacelike to that of $\varrho_{1}, \varrho_{2}$ and $\varrho_{2}^{\prime}$, the support of $\varrho_{2}^{\prime}$ is spacelike to that of $\varrho_{1}, \varrho_{2}$ and $\varrho_{1}^{\prime}$ and the support of $\varrho_{1}^{\prime} \varrho_{2}^{\prime}$ is spacelike to that of $\varrho_{1} \varrho_{2}$. Then by definition, $\varepsilon_{\varrho_{1}}=U_{1}^{-1} \varrho_{1}\left(U_{1}\right), \varepsilon_{\varrho_{2}}=U_{2}^{-1} \varrho_{2}\left(U_{2}\right)$ and $\varepsilon_{\varrho_{1} \varrho_{2}}=\varrho_{1}\left(U_{2}\right)^{-1} U_{1}^{-1} \varrho_{1} \varrho_{2}\left(U_{1} \varrho_{1}\left(U_{2}\right)\right)$. By the special choice of support regions we have

$$
\varrho_{1}^{\prime}\left(U_{2}\right)=U_{2} ; \quad \varrho_{2}^{\prime}\left(U_{1}\right)=U_{1} ; \quad \Lambda_{1} U_{2}=U_{2} \varrho_{2}\left(\Lambda_{1}\right),
$$

where we write $\Lambda_{i}=\phi_{i}\left(\varepsilon_{Q_{2}}\right), i=1,2$. Then

$$
\phi_{2} \phi_{1}\left(\varepsilon_{\varrho_{1} \varrho_{2}}\right)=\phi_{2}\left(U_{2}^{-1} \phi_{1}\left(U_{1}^{-1}\right)\right) U_{1} \varrho_{1}\left(U_{2}\right)=\phi_{2}\left(U_{2}^{-1} \Lambda_{1} U_{1}^{-1}\right) U_{1} \varrho_{1}\left(U_{2}\right)
$$

and using (6.18) repeatedly we find

$$
\phi_{2} \phi_{1}\left(\varepsilon_{\varrho_{1} \varrho_{2}}\right)=\Lambda_{1} U_{1}^{-1} \Lambda_{2} U_{1}=\Lambda_{1} \varrho_{1}\left(\Lambda_{2}\right) \text { giving (6.17). }
$$

6.8. Corollary. a) If $\phi_{1}$ and $\phi_{2}$ are standard then $\phi_{2} \phi_{1}$ is standard.

b) The product of sectors with finite statistics has finite statistics.

c) If $\varrho_{1}, \varrho_{2} \in \Delta_{t}$ have finite statistics and $\varrho=\varrho_{1} \varrho_{2}$ then

$$
\begin{aligned}
& b(\varrho)=b\left(\varrho_{1}\right) b\left(\varrho_{2}\right)+f\left(\varrho_{1}\right) f\left(\varrho_{2}\right) \\
& f(\varrho)=b\left(\varrho_{1}\right) f\left(\varrho_{2}\right)+f\left(\varrho_{1}\right) b\left(\varrho_{2}\right) \quad d(\varrho)=d\left(\varrho_{1}\right) d\left(\varrho_{2}\right) .
\end{aligned}
$$

The unitary invariants $b, d$ and $f$ provide just the information needed to characterize the statistics.

6.9. Theorem. Let $\varrho \in \Delta_{t}$ then the Young tableaux associated with the representations $\varepsilon_{Q}^{(n)}$ of $P^{(n)}, n \geqq 1$ are all Young tableaux if $d(\varrho)=\infty$ and otherwise all Young tableaux with at most $b(\varrho)$ rows of length $>f(\varrho)$ and at most $f(\varrho)$ columns of length $>b(\varrho)$.

To prove this theorem we must generalize Lemma 5.1, Proposition 5.2 and Lemma 5.4. We content ourselves here with stating the analogue of Proposition 5.2 which should provide enough guidance for the interested reader. 
6.10. Proposition. Let $\phi$ be a standard left inverse for a $\varrho \in \Delta_{t}$ with finite statistics, then

$$
\phi^{n}\left(\varepsilon_{\varrho}^{(n)}(p)\right)=\omega(p) I, \quad p \in P^{(n)}
$$

where $\omega$ is the trace state on $P^{(n)}$ multiplicative on disjoint cycles and taking the value $d(\varrho)^{-2 k}(b(\varrho)-f(\varrho))$ on a $2 k$-cycle and $d(\varrho)^{-2 k}$ on a $2 k+1$-cycle. The cyclic representation of $P^{(n)}$ generated by $\omega$ is quasiequivalent to $\varepsilon_{\varrho}^{(n)}$.

Of course by Corollary $6.8 \mathrm{a}, \phi^{n}$ is a standard left inverse for $\varrho^{n}$ so that comparing with (6.14) we get

$$
\omega(p)=\operatorname{Tr}_{\varrho^{n}}\left(\varepsilon_{\varrho}^{(n)}(p)\right)
$$

which at least proves that $\omega$ is a trace state. However, we refrain from giving further details.

\section{Appendix}

Suppose $\pi$ is a representation of $\mathfrak{A}$ and $V$ a unitary operator from $\mathscr{H}_{0}$ onto $\mathscr{H}_{\pi}$ such that, for a given double cone $\mathcal{O}$,

$$
\pi(A)=V A V^{-1} \text { for } A \in \mathfrak{A}\left(\mathcal{O}^{\prime}\right) .
$$

Let $\omega$ be a normal state in the representation $\pi$; we want to prove that $\omega$ fulfills the relation (1.3) of the introduction:

$$
\lim _{n \rightarrow \infty}\left\|\left.\left(\omega-\omega_{0}\right)\right|_{\hat{o}_{n}^{\prime}}\right\|=0
$$

where the sequence of double cones $\mathcal{O}_{n}$ exhausts space-time in the limit $n \rightarrow \infty$ (cf. (1.2)).

The argument is the same as in [13] and we give it here for the convenience of the reader. Suppose that (A.2) is incorrect: there exists a subsequence $\mathcal{O}_{n_{i}}$ and operators $B_{i}$ from the unit ball of $\mathfrak{A}\left(\mathcal{O}_{n_{i}}^{\prime}\right)$ such that for all $i=1,2, \ldots$

$$
\left|\left(\omega-\omega_{0}\right)\left(B_{i}\right)\right|>\varepsilon
$$

where $\varepsilon$ is a positive number.

Since the unit ball of $\mathscr{B}\left(\mathscr{H}_{0}\right)$ is weakly compact, the sequence $B_{i}$ has a weak limit point $B$. The operator $B$ must in fact be equal to a complex number $c$ times the identity operator on $\mathscr{H}_{0}$ since

$$
B \in\left\{\bigcup_{i} \mathfrak{A}\left(\mathcal{O}_{n_{i}}\right)\right\}^{\prime}=\mathfrak{A}^{\prime}=C I .
$$

In particular $B \in \mathfrak{A}\left(\mathcal{O}_{n_{i}}^{\prime}\right)$. For sufficiently large $i, \mathcal{O}$ is included in $\mathcal{O}_{n_{i}}$ and by Eq. (A.1), $\omega$ is normal on $\mathfrak{A}\left(\mathcal{O}_{n_{i}}^{\prime}\right)$, i.e., weakly continuous on its unit ball; hence by (A.3) we find

$$
\left|\left(\omega-\omega_{0}\right)(B)\right|>\varepsilon .
$$


However, $B=c I$ and $\omega(B)=\omega_{0}(B)=c$; the contradiction proves that (A.2) is a consequence of (A.1).

The statement above admits a partial converse under some restrictive hypothesis on the representation $\pi$. For each double cone $\mathcal{O}$ let $\mathfrak{\Im}_{\pi}(\mathcal{O})$ denote the von Neumann algebra $\pi\left(\mathfrak{U}\left(\mathcal{U}^{\prime}\right)\right)^{\prime}$.

As we shall see below, the following property (the generalization of property $B$ to the representation $\pi$ ) can be derived in the relevant cases:

Let $\mathcal{O}$ be a double cone included in the interior of the double cone $\mathcal{O}_{1}$. If $E \in \Im_{\pi}(\mathcal{O})$ is a non-zero projection, there is an isometry $W \in \mathfrak{S}_{\pi}\left(\mathcal{O}_{1}\right)$ such that $W W^{*}=E, W^{*} W=I$.

This means the following: take any subrepresentation of $\pi \mid \mathfrak{A}\left(\mathcal{O}^{\prime}\right)$ acting on a subspace $\mathscr{H}_{1} \subset \mathscr{H}_{\pi}$ and restrict it to $\mathfrak{U}\left(\mathcal{O}_{1}^{\prime}\right)$ then the resulting representation is unitarily equivalent to $\pi \mid \mathfrak{U}\left(\mathcal{O}_{1}^{\prime}\right)$. In fact the orthogonal projection $E$ onto $\mathscr{H}_{1}$ belongs to $\pi\left(\mathfrak{A}\left(\mathcal{O}^{\prime}\right)\right)^{\prime}=\mathfrak{S}_{\pi}(\mathcal{O})$ hence there is an isometry $W$ as in (A.4) commuting with $\pi\left(\mathfrak{Q}\left(\mathcal{O}_{1}^{\prime}\right)\right) ; W$ realizes the above equivalence.

Note also that if $\pi_{1}$ and $\pi_{2}$ satisfy (A.4) and the representations $\pi_{1} \mid \mathfrak{A}\left(\mathcal{O}^{\prime}\right)$ and $\pi_{2} \mid \mathfrak{U}\left(\mathcal{O}^{\prime}\right)$ are not disjoint (in other words a non-zero subrepresentation of the first is unitarily equivalent to a subrepresentation of the second) it follows that $\pi_{1} \mid \mathfrak{A}\left(\mathcal{O}_{1}^{\prime}\right)$ and $\pi_{2} \mid \mathfrak{H}\left(\mathcal{O}_{1}^{\prime}\right)$ are unitarily equivalent for any double cone $\mathcal{O}_{1}$ containing $\mathcal{O}$ in its interior.

A.1. Proposition. Let $\omega$ be a state of $\mathfrak{A}$ such that

$$
\lim _{n \rightarrow \infty}\left\|\left.\left(\omega-\omega_{0}\right)\right|_{O_{n}^{\prime}}\right\|=0
$$

for an increasing sequence of double cones $\mathcal{O}_{n}$. If the GNS representation $\pi_{\omega}$ fulfills (A.4), there is a double cone $\mathcal{O}$ such that

$$
\pi_{\omega}\left|\mathfrak{A}\left(\mathcal{O}^{\prime}\right) \cong \pi_{0}\right| \mathfrak{A}\left(\mathcal{O}^{\prime}\right) .
$$

Proof. For sufficiently large $n_{0}$ we have

$$
\left\|\left.\left(\omega-\omega_{0}\right)\right|_{\hat{O}_{0}^{\prime}}\right\|<2
$$

and, by a theorem of Glimm and Kadison [14], the states $\omega \mid \mathfrak{A}\left(\mathcal{O}_{n_{0}}^{\prime}\right)$ and $\omega_{0} \mid \mathfrak{A}\left(\mathscr{O}_{n_{0}}^{\prime}\right)$ induce non-disjoint representations of $\mathfrak{A}\left(\mathcal{O}_{n_{0}}^{\prime}\right)^{16}$. These representations are subrepresentations of $\pi_{\omega} \mid \mathfrak{U}\left(\mathcal{O}_{n_{0}}^{\prime}\right)$ and $\pi_{0} \mid \mathfrak{A}\left(\mathcal{O}_{n_{0}}^{\prime}\right)$ respectively, hence a fortiori the latter are not disjoint. If the double cone

${ }^{16}$ If the representations $\pi_{\omega_{1}}$ and $\pi_{\omega_{2}}$ induced by the states $\omega_{1}$ und $\omega_{2}$ over a $C^{*}$-algebra $\mathfrak{B}$ are disjoint, the von Neumann algebra $\pi_{\omega_{1}} \oplus \pi_{\omega_{2}}(\mathfrak{B})^{\prime \prime}$ coincides with $\pi_{\omega_{1}}(\mathfrak{B})^{\prime \prime} \oplus \pi_{\omega_{2}}(\mathfrak{B})^{\prime \prime}$ hence contains the operator $B=I \oplus-I$. The form $\omega_{1}-\omega_{2}$ over $\pi_{\omega_{1}} \oplus \pi_{\omega_{2}}(\mathfrak{B})$ has a unique ultraweakly continuous extension $\psi$ to $\pi_{\omega_{1}} \oplus \pi_{\omega_{2}}(\mathfrak{B})^{\prime \prime}$ with the same norm, and $2 \geqq\left\|\omega_{1}-\omega_{2}\right\|$ $=\|\psi\| \geqq|\psi(B)|=2$ so $\left\|\omega_{1}-\omega_{2}\right\|=2$. 
$\mathcal{O}$ contains $\mathcal{O}_{n_{0}}$ in its interior, it follows by the remarks above that

$$
\pi_{\omega}\left|\mathfrak{A}\left(\mathcal{O}^{\prime}\right) \cong \pi_{0}\right| \mathfrak{U}\left(\mathcal{O}^{\prime}\right)
$$

We now discuss how property (A.4) is related to more standard assumptions which may be expected to hold for observables derived from an underlying field theory. The observable algebra is required to be invariant under space-time translations and to satisfy a so-called additivity property:

(i) There is a continuous unitary representation $x \rightarrow U_{0}(x)$ of the space-time translation group, acting on $\mathscr{H}_{0}$ such that for each $\mathcal{O} \in \mathscr{K}$, $A \in \mathfrak{A}(\mathcal{O})$ and $x \in \boldsymbol{R}^{4}$

$$
U_{0}(x) A U_{0}(x)^{-1} \equiv \alpha_{x}(A) \in \mathfrak{A}(\mathcal{O}+x)
$$

(ii) If $\mathcal{O}_{1}, \ldots, \mathcal{O}_{n}$ are double cones covering the double cone $\mathcal{O}$, the von Neumann algebra generated by the $\mathfrak{A}\left(\mathcal{O}_{i}\right), i=1,2, \ldots, n$, includes $\mathfrak{A}(\mathcal{O})$.

If $\pi$ is a locally normal representation of $\mathfrak{A}$, the algebras $\pi(\mathfrak{U}(\mathcal{O}))$ are von Neumann algebras and satisfy the additivity property analogous to (ii). Hence for any double cone $\mathcal{O}$ the collection of algebras $\pi(\mathfrak{A}(\mathcal{O}+x))$, $x$ varying over space-time, generates $\pi(\mathfrak{l})^{\prime \prime}$ as a von Neumann algebra. Therefore a Theorem of Borchers [4, Theorem III.3] implies that an irreducible locally normal representation $\pi$ fulfills (A.4) whenever it satisfies the following spectrum condition:

There exists a continuous unitary representation $x \rightarrow U(x)$ of the space-time translation group, acting on $\mathscr{H}_{\pi}$, such that

(a) $\pi\left(\alpha_{x}(A)\right)=U(x) \pi(A) U(x)^{-1}, x \in \boldsymbol{R}^{4}, A \in \mathfrak{A}$;

(b) the spectrum of $U$ is contained in the forward light cone.

It will be shown elsewhere that if $\varrho \in \Delta_{t}$ leads to a sector with finite statistics and covariant under the Poincaré group, the conjugate representation $\pi$ described in section III is also covariant, and both $\pi$ and $\varrho$ fulfill the spectrum condition as a consequence of the positivity of the energy in the vacuum sector. Under the additivity assumption (ii) for the observable algebra, the conjugate representation will then be in the family described in the introduction.

Acknowledgments. Our collaboration in this work during the past two years was made possible through the help of several institutions. We gratefully acknowledge the hospitality and support extended to us at various stages by the Centre Universitaire de MarseilleLuminy, the CNRS, the DRME (contract 46/68), the Department of Mathematics of the University of Pennsylvania, the NSF, the Air Force Office of Scientific Research under Contract No. F 44620-71-C-0108 and the Courant Institute for Mathematical Sciences. We also would like to give warm thanks to our collegues D. Kastler, R. V. Kadison, J. Glimm and A. S. Wightman for their concern and help. 
230 S. Doplicher, R. Haag, and J. E. Roberts: Local Observables and Particle Statistics I

\section{References}

1. Doplicher, S., Haag, R., Roberts, J.E.: Fields, observables and gauge transformations I. Commun. math. Phys. 13, 1-23 (1969).

2. - - Fields, observables and gauge transformations II. Commun. math. Phys. 15, 173-200 (1969).

3. Wick, G.C., Wightman,A.S., Wigner,E.P.: The intrinsic parity of elementary particles. Phys. Rev. 88, $101-105$ (1952).

4. Borchers, H. J.: A remark on a theorem of B. Misra. Commun. math. Phys. 4, 315-323 (1967).

5. Stolt, R. A., Taylor, J. R.: Classification of paraparticles. Phys. Rev. D, 1, 2226-2228 (1970).

6. Hartle,J.B., Stolt, R.A., Taylor,J.R.: Paraparticles of infinite order. Phys. Rev. D2, 1759-1760 (1970).

7. Greenberg, O.W., Messiah,A.M.L.: Symmetrization postulate and its experimental formulation. Phys. Rev. 136, B248-267 (1964).

8. Drühl,K., Haag, R., Roberts,J.E.: On parastatistics. Commun. math. Phys. 18, 204-226 (1970).

9. Kadison, R.V.: The trace in finite operator algebras. Proc. Amer. Math. Soc. 12, 973-977 (1961).

10. Stinespring, W.F.: Positive functions on $C^{*}$-algebras. Proc. Amer. Math. Soc. 6, 211-216 (1955).

11. Eilenberg, S., Kelly, G.M.: Closed categories. In: Proceedings of the Conference on Categorical Algebra, La Jolla 1965. Berlin, Heidelberg, New York: Springer 1966.

12. Weyl,H.: The classical groups. Princeton: Princeton University Press 1946.

13. Powers, R.T.: Representations of uniformly hyperfinite algebras and their associated von Neumann rings. Ann. Math. 86, 138-171 (1967).

14. Glimm,J., Kadison, R.V.: Unitary operators in $C^{*}$-algebras. Pacific J. Math. 10, 547-556 (1960).

S. Doplicher

Instituto di Fisica

„Guglielmo Marconi“

Piazzale delle Scienze 5

Roma
R. Haag

J. E. Roberts

II. Institut für Theoretische Physik der Universität Hamburg

D-2000 Hamburg 50, Luruper Chaussee 149 